\title{
Distinct neural progenitor pools in the ventral telencephalon generate diversity in striatal spiny projection neurons
}

\author{
Fran van Heusden ${ }^{1,3}$, Anežka Macey-Dare ${ }^{1,3}$, Rohan N. Krajeski ${ }^{1}$, Andrew Sharott ${ }^{2}$, \\ Tommas Jan Ellender ${ }^{1 *}$ \\ ${ }^{1}$ Department of Pharmacology, OX1 3QT, Oxford \\ ${ }^{2}$ MRC BNDU, OX1 3TH, Oxford \\ ${ }^{3}$ Contributed equally \\ * Corresponding author \\ Tommas Ellender \\ Department of Pharmacology \\ Mansfield Road, OX1 3QT \\ Oxford, United Kingdom \\ tommas.ellender@pharm.ox.ac.uk
}

Number of pages:

42

Number of Figures:

Number of Tables:

5

Number of Supplemental Figures: 3

Wordcount Abstract:

Wordcount Introduction:

Wordcount Results:

Wordcount Discussion:

Keywords: basal ganglia, striatum, development, embryonic neural progenitors, spiny projection neurons, apical intermediate progenitors, neural circuits

Running title: Fate mapping of striatal neural progenitors.

Conflict of interest: We declare no conflict of interest.

Acknowledgements: We would like to thank members of the Ellender lab for advice and comments. We gratefully acknowledge Ulrich Müller and Tarik Haydar for providing reagents, Peter Somogyi, Peter Magill and Colin Akerman for providing access to equipment, Ben Micklem for providing technical assistance and Monzilur Rahman, Rebecca Waterfield, Nicholas Pasternack and Eoin Mac Reamoinn for initial help. TJE was supported by a MRC Career Development Award (MR/M009599/1) and AMD by an Imperial College research bursary.

Author Contributions: FvH, AMD and TJE designed the experiments. FvH and TJE performed the electrophysiology experiments and analysis. FvH, AMD, RNK and AS performed anatomical experiments and analysis. All authors discussed the data. TJE wrote the manuscript. No competing interests for any of the authors. 


\section{7}

48

49

50

\section{Abstract}

Heterogeneous populations of neural progenitors in the embryonic lateral ganglionic eminence (LGE) generate all GABAergic spiny projection neurons (SPNs) found in the striatum. Here we investigate how this diversity in neural progenitors relates to diversity of adult striatal neurons and circuits. Using a combination of in utero electroporation to fluorescently pulse-label striatal neural progenitors in the LGE, brain slice electrophysiology, electrical and optogenetic circuit mapping and immunohistochemistry, we characterise a population of neural progenitors enriched for apical intermediate progenitors (aIPs) and a distinct population of other progenitors (OPs) and their neural offspring. We find that neural progenitor origin has subtle but significant effects on the properties of striatal SPNs. Although aIP and OP progenitors can both generate D1-expressing direct pathway as well as D2-expressing indirect pathway SPNs found intermingled in the striatum, the aIP derived SPNs are found in more medial aspects of the striatum, exhibit more complex dendritic arbors with higher spine density and differentially sample cortical input. Moreover, optogenetic circuit mapping of the aIP derived neurons show that they further integrate within striatal circuits and innervate both local D1 and D2 SPNs. These results show that it is possible to fluorescently pulse-label distinct neural progenitor pools within the LGE and provide evidence that neural progenitor heterogeneity can contribute to the diversity of striatal SPNs. 


\section{Introduction}

A fundamental question in neuroscience is how neuronal cell types and neural circuits arise and what critically guides their development. Recent studies in the dorsal telencephalon or pallium have highlighted important and distinct roles for embryonic neural progenitors, at both the level of single neural progenitors ( $\mathrm{Yu}$ et al., 2009; $\mathrm{Yu}$ et al., 2012; Cadwell et al., 2019) and distinct pools of neural progenitors (Tyler et al., 2015; Ellender et al., 2018), in shaping neuronal identity and synaptic connectivity. However, much less is known about how embryonic neural progenitors in the ventral telencephalon or subpallium contribute to the cellular diversity and neural circuitry found within ventral brain structures. Neural progenitors in the ventral portions of the embryonic brain can be found in the ganglionic eminences - transitory structures that generate most interneurons of the brain and the spiny projection neurons (SPNs) of the striatum (Wonders \& Anderson, 2006; Wamsley \& Fishell, 2017). In this study we focused on the neural progenitors found within the lateral ganglionic eminence (LGE) (Graybiel \& Ragsdale, 1978; Olsson et al., 1998; Mason et al., 2005; Pilz et al., 2013; Kelly et al., 2018), which give rise to the striatal SPNs, and include radial glial cells, basal radial glial cells, subapical progenitors, basal progenitors and short neural precursors, amongst others (Olsson et al., 1998; Stenman et al., 2003; Pilz et al., 2013). Many of these are not unique to the LGE and have previously been characterised in detail in the proliferative zones of the cortex (Noctor et al., 2001; Noctor et al., 2004; Gal et al., 2006; Kowalczyk et al., 2009; Stancik et al., 2010; Shitamukai et al., 2011; Wang et al., 2011; Franco \& Muller, 2013; Taverna et al., 2014). However, the relative abundance as well as key proliferative behaviours of many of these progenitors differ between cortex and the LGE (Pilz et $a l ., 2013)$. Furthermore, it is unknown to what extent these different pools of neural progenitors contribute to the diversity of striatal projection neurons.

To investigate the relationship between neural progenitor pool and striatal neuron diversity, we used in utero electroporation to fluorescently pulse-label two pools of actively dividing neural progenitors in the LGE distinguished by their differential expression of the tubulin alpha1 (Ta1) promoter (Gal et al., 2006; Stancik et al., 2010). We find that Tal-expressing neural progenitors exhibit many of the characteristics of the previously described short neural precursors and subapical progenitors found within the LGE (Pilz et al., 2013), including division in the 
104 ventricular zone, lack of basal processes during division and relatively fast cell-cycle

105 kinetics. In contrast, the non-Tal-expressing neural progenitors tend to retain their 106 basal processes during division and exhibit slower cell-cycle kinetics. Overall, our 107 observations suggest that T $\alpha 1$-expressing neural progenitors consist of intermediate 108 progenitors found in apical regions of the LGE such as short neural precursors and 109 subapical progenitors (Pilz et al., 2013) and we therefore refer to them collectively as 110 apical intermediate progenitors (aIPs) and the non-T $\alpha 1$-expressing neural progenitors 111 consist of all other neural progenitors that are actively dividing at the same time and 112 we collectively refer to them as other progenitors (OPs). We find that aIP and OP 113 neural progenitors mainly generate striatal neurons, with all the hallmarks of 114 GABAergic spiny projection neurons (SPNs) including expression of neurochemical 115 markers such as CTIP2, but also generate a small number of neurons found within the 116 olfactory bulb. Using the neurochemical marker PPE we find that both aIP and OP 117 neural progenitors generate D1-expressing direct pathway as well as D2-expressing 118 indirect pathway SPNs. Stereological investigation of progenitor derived striatal 119 neurons shows that aIP derived neurons are on average found in more medial aspects 120 of the striatum. Whole-cell patch-clamp recordings of aIP and OP derived neurons in 121 acute striatal slices reveals that their electrical properties are similar and consistent 122 with those of SPNs. Indeed, subsequent anatomical reconstruction of recorded 123 neurons confirms that they exhibit all the hallmarks of SPNs, including radially 124 oriented dendrites with large numbers of spines. Interestingly, we find differences 125 with aIP derived SPNs exhibiting a greater local dendritic complexity as well as a 126 higher density of spines. Lastly, using both electrical and optogenetic circuit mapping 127 we find that both aIP and OP derived SPNs integrate within striatal circuits. Firstly, 128 both receive excitatory glutamatergic input from cortex with aIP derived SPNs 129 exhibiting a significantly prolonged response to cortical activation. Secondly, aIP 130 derived SPNs form local GABAergic inhibitory synaptic connections with 131 neighbouring D1 and D2 SPNs.

132 In conclusion, this study shows it is possible to label distinct pools of neural 133 progenitors in the LGE and suggests that neural progenitor heterogeneity contributes 134 to striatal diversity in that neural progenitor origin has subtle but significant effects on 135 the spatial distribution of striatal neurons, their morphology as well as their sampling 136 of excitatory inputs. 
138

139

140

141

142

143

144

145

146

147

148

149

150

151

152

153

154

155

156

157

158

159

160

161

162

163

164

165

166

167

168

169

170

171

\section{Results}

The LGE of the ventral telencephalon contains tubulin alpha1 (Ta1) expressing neural progenitors

To investigate the relationship between the diverse pools of neural progenitors in the LGE and their contribution to striatal neuronal identity and connectivity we performed in utero electroporation (IUE) to fluorescently label actively dividing progenitors in the ventricular zone (Stancik et al., 2010). Two DNA constructs were electroporated into the LGE: a T $\alpha 1$-cre construct in which cre recombinase is under the control of a portion of the T $\alpha 1$ promoter (Stancik et al., 2010), and a C $\beta A-F L E x$ reporter construct that incorporates a flexible excision (FLEx) cassette where cre recombination permanently switches expression from TdTomato fluorescent protein to enhanced green fluorescent protein (GFP) (Franco et al., 2012).

We find that 24 hours after IUE the LGE contains both GFP and TdTomato expressing cells, consisting of T $\alpha 1$-expressing $\left(\mathrm{T} \alpha 1^{+}\right)$and non-T $\alpha 1$-expressing $\left(\mathrm{T} \alpha 1^{-}\right)$ neural progenitors and young migrating neurons (Figure 1A). We find that the time of IUE influenced the relative proportion of GFP and TdTomato expressing cells seen 24 hours later, with IUE at E15.5 resulting in a greater number of $\mathrm{T} \alpha 1^{+} / \mathrm{GFP}^{+}$cells (E12.5: $4.8 \pm 4.1 \%$ and E15.5: $34.5 \pm 3.0 \%$, Mann-Whitney test, $\mathrm{p}=0.002, \mathrm{n}=7$ and 17 embryonic brains, Figure 1B), suggesting that, similar to their cortical counterparts (Stancik et al., 2010; Ellender et al., 2018) Ta1-expressing progenitors form a considerable population of actively proliferating cells during later periods of neurogenesis.

Using IUE at E15.5 we next investigated the spatial distribution of $\mathrm{T} \alpha 1^{+} / \mathrm{GFP}^{+}$ and $\mathrm{T} \alpha 1^{-} / \mathrm{TdTomato}^{+}$cells within the LGE to further characterize these cells and gain insight into their proliferative behaviour. Firstly, we assessed the location of all $\mathrm{T} 1^{+} / \mathrm{GFP}^{+}$and $\mathrm{T} \alpha 1^{-} / \mathrm{TdTomato}^{+}$cells and dividing progenitors relative to the ventricular wall 24 hours after IUE (Figure 1C and D). $\mathrm{T} 1^{+} / \mathrm{GFP}^{+}$and $\mathrm{T} \alpha 1^{-}$ $/$ TdTomato $^{+}$cells can be found interspersed in the proliferative zone of the LGE with the $\mathrm{T} 1^{+} / \mathrm{GFP}^{+}$labeled cells on average significantly further away from the ventricular wall than the $\mathrm{T} \alpha 1^{-} / \mathrm{TdTomato}^{+}$labeled cells $\left(\mathrm{T} \alpha 1^{+} / \mathrm{GFP}^{+}: 66.24 \pm 3.57 \mu \mathrm{m}\right.$

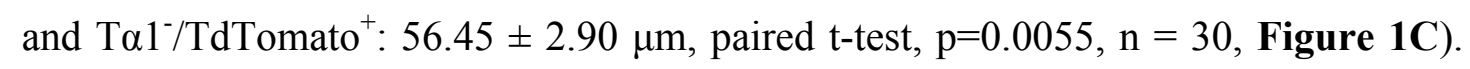
However, when we limited analysis to only actively dividing progenitor cells, labeled 
with the mitotic marker phospho-histone $\mathrm{H} 3 \mathrm{(pH3)}$, no difference was seen in their distance from the ventricle $\left(\mathrm{T} \alpha 1^{+} / \mathrm{GFP}^{+}: 52.28 \pm 6.21 \mu \mathrm{m}\right.$ and $\mathrm{T} \alpha 1^{-} / \mathrm{TdTomato}^{+}: 48.78$ $\pm 3.99 \mu \mathrm{m}, \mathrm{p}=0.586$, paired t-test, $\mathrm{n}=16$ brains, Figure 1D). These results are

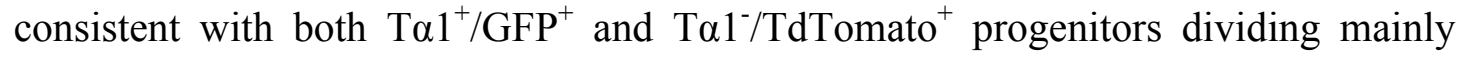
within the ventricular zone of the LGE (Pilz et al., 2013). Furthermore, our results suggest that 24 hours after IUE T $\alpha 1^{+} / \mathrm{GFP}^{+}$cells, likely consisting of young migrating neurons and non-dividing neural progenitors, inhabit increasingly subapical positions within the LGE. Whereas in the cortex young migrating neurons mostly exhibit radial migration (Noctor et al., 2001) newly born neurons in the striatum undergo tangential, radial and other forms of migration (Halliday \& Cepko, 1992; Tan \& Breen, 1993; Reid \& Walsh, 2002; Tinterri et al., 2018). Indeed, when we look at the average dispersion of all labeled cells in a subset of brain slices along two axis perpendicular to the ventricular wall we find a positive linear correlation between the amount of horizontal and vertical spread of labeled cells $(\mathrm{R}=0.56, \mathrm{p}=0.00004, \mathrm{n}=26$ slices $)$ which is similar for both $\mathrm{T} 1^{+} / \mathrm{GFP}^{+}$and $\mathrm{T}^{-} 1^{-} / \mathrm{TdTomato}^{+}$cells $(\mathrm{p}>0.05)$ and is not seen when we limit the analysis to only actively dividing neural progenitors $(R=0.24$, $\mathrm{p}=0.18, \mathrm{n}=20$ slices, Figure 1E). Lastly, the LGE has also been shown to consist of several domains depending on differential expression of certain transcription factors (Stenman et al., 2003; Flames et al., 2007; Xu et al., 2018). When we split the LGE in 4 different domains we find that both $\mathrm{T} 1^{+} / \mathrm{GFP}^{+}$and $\mathrm{T} \alpha 1^{-} / \mathrm{TdTomato}^{+}$cells can be found in all progenitor domains of the LGE (Supplemental Figure 1).

We next investigated the morphology of actively dividing neural progenitors in the LGE. The distinguishing features of short neural precursors in the dorsal and ventral telencephalon are their division at the ventricular wall and their short morphology during division lacking a prominent basal process contacting pial surfaces (Gal et al., 2006; Stancik et al., 2010; Pilz et al., 2013; Ellender et al., 2018). Indeed, when we look at the morphology of labeled neural progenitors we find that many actively dividing $\mathrm{T} \alpha 1^{+} / \mathrm{GFP}^{+}$progenitors exhibit a short rounded morphology during division and could be found at the ventricular wall (Figure 1F). However, some $\mathrm{T} 1^{+} / \mathrm{GFP}^{+}$progenitors divide slightly away from the ventricular wall resembling subapical progenitors (Pilz et al., 2013) (Figure 1F). In contrast, many

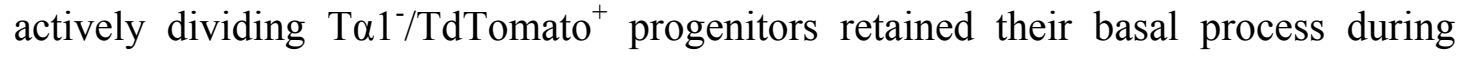
division and could be found either at or away from the ventricular wall (Figure 1G). This was confirmed quantitatively by assessment of the number of cells in the VZ that 
retained a basal process. Overall, we find that $\mathrm{T} \alpha 1^{+} / \mathrm{GFP}^{+}$cells often lacked a basal

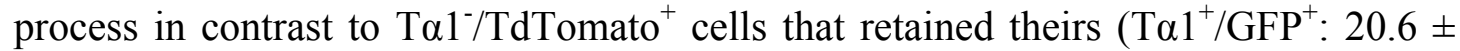

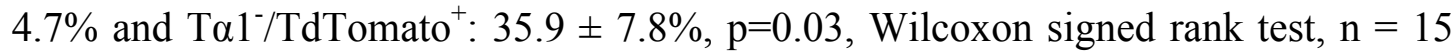
brains, Figure 1H).

A further distinguishing characteristic of both short neural precursors and subapical progenitors found in the LGE is their relatively fast cell cycle kinetics (Pilz et al., 2013). To investigate whether the $\mathrm{T} 1^{+} / \mathrm{GFP}^{+}$progenitor population exhibits fast cell cycle durations we performed IUE at E15.5 and subsequently fixed tissue at varying delays ranging from 12 to 26 hours and labeled dividing neural progenitors with the mitotic marker pH3 (Stancik et al., 2010). Consistent with the fast cell cycle kinetics described for the short neural precursors and subapical progenitors in the LGE we find that the $\mathrm{T} \alpha 1^{+} / \mathrm{GFP}^{+}$progenitors return to G1/S phase faster that the T $\alpha 1^{-}$ $/$ TdTomato $^{+}$progenitors. Indeed, after a 16 hour delay $\mathrm{T} \alpha 1^{+} / \mathrm{GFP}^{+}$progenitors are the dominant population of $\mathrm{pH} 3$ expressing cells $\left(\mathrm{T} \alpha 1^{+} / \mathrm{GFP}^{+}: 19.85 \pm 7.15 \%\right.$ and $\mathrm{T} \alpha 1^{-}$ TdTomato $^{+}: 5.18 \pm 1.72 \%$, t-test, $\mathrm{p}=0.025, \mathrm{n}=6$ brains) whereas after a 20 hour delay it is the $\mathrm{T} 1^{-} / \mathrm{TdTomato}^{+}$progenitors which form the dominant population of pH3 expressing cells $\left(\mathrm{T} \alpha 1^{+} / \mathrm{GFP}^{+}: 4.98 \pm 2.11 \%\right.$ and $\mathrm{T}^{2} 1^{-} / \mathrm{TdTomato}^{+}: 15.81 \pm$ $3.63 \%$, t-test, $\mathrm{p}=0.036, \mathrm{n}=8$ brains, Figure 1I).

In conclusion, our results suggest that the LGE in the ventral telencephalon contains a population of Tal-expressing neural progenitors that share many characteristics with short neural precursors and subapical progenitors previously described (Pilz et al., 2013), including a ventricular location of division, a short round morphology during division and fast cell-cycle kinetics. In contrast we are also able to label a population of neural progenitor that does not express $\mathrm{T} \alpha 1$ that share many characteristics with radial glial cells (Pilz et al., 2013) and which tend to retain its basal process and exhibits slower cell cycle kinetics. 
A
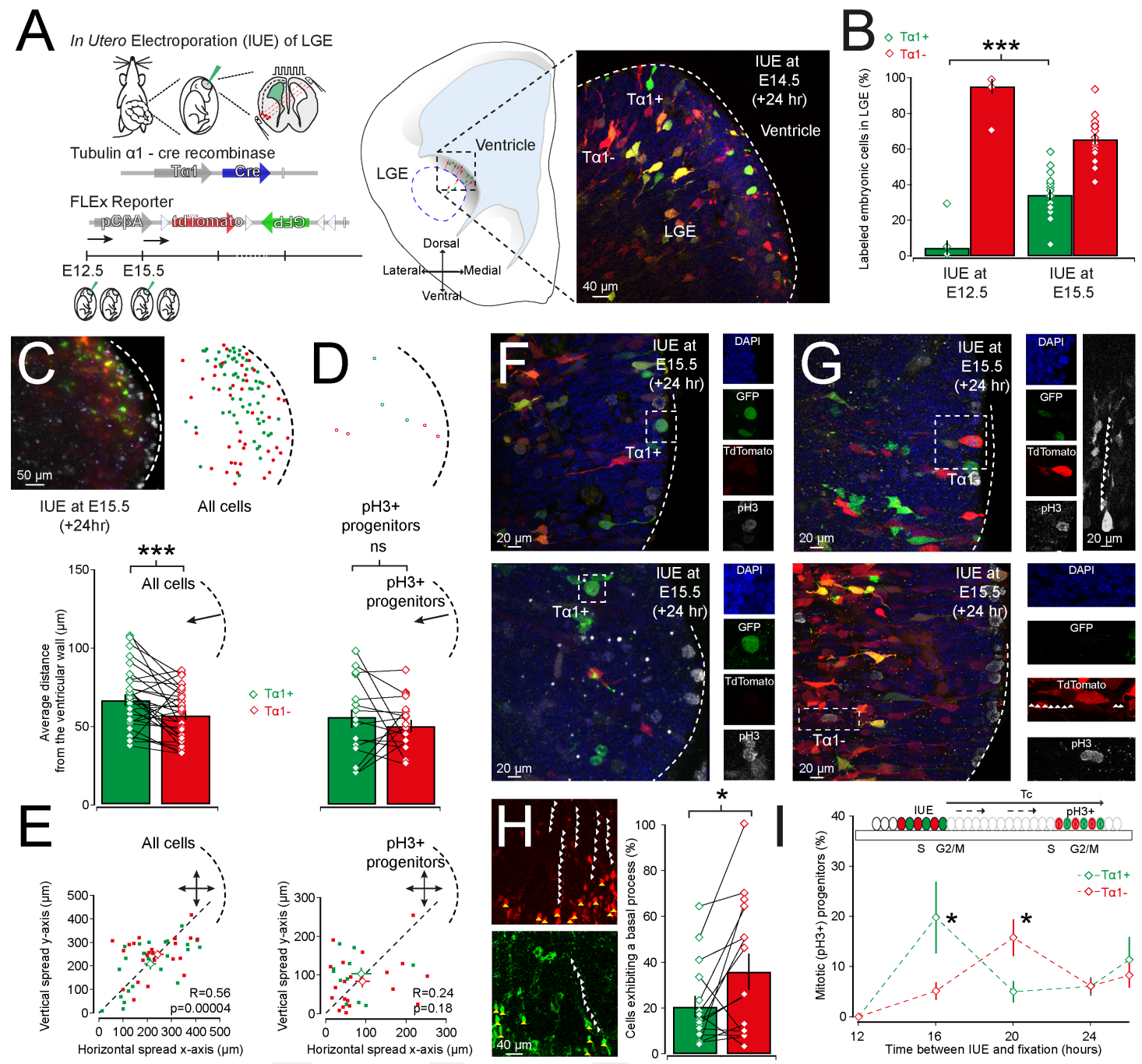

Figure 1: The LGE contains both Ta1-expressing and non-Ta1-expressing neural progenitors. (A) In utero electroporation (IUE) of Ta1-cre and FLEx reporter plasmids was performed at embryonic day (E)12.5 and E15.5 to label Ta1-positive $\left(\mathrm{T} \alpha 1^{+}\right)$and $\mathrm{T} \alpha 1$-negative $\left(\mathrm{T} \alpha 1^{-}\right)$neural progenitors lining the ventricular wall of the LGE with respectively the fluorescent markers GFP or TdTomato (left). 24 hours


and young neurons can be found in the LGE (right). Yellow cells were were assumed to have undergone recombination relatively recently and counted as $\mathrm{GFP}^{+}$.

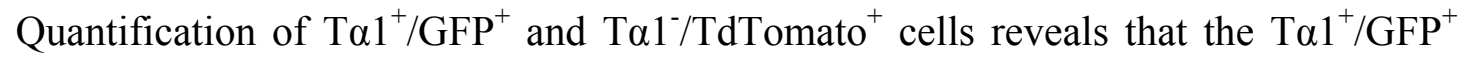
cells form a substantial population during late stages of embryogenesis. (C) The distance from the ventricle of ${\mathrm{T} \alpha 1^{+} / \mathrm{GFP}^{+} \text {and } \mathrm{T} \alpha 1^{-} / \mathrm{TdT} \text { Tomato }}^{+}$cells 24 hours after

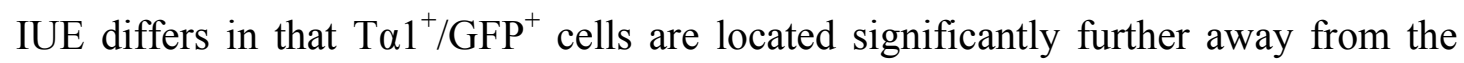
ventricular wall. (D) A difference in distance from the ventricle is not seen for

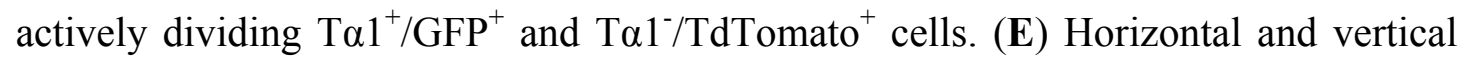
migration of all labeled cells exhibits a linear correlation (left) whereas this is not the case for actively dividing progenitors (right). (F) Actively dividing $\mathrm{T} \alpha 1^{+} / \mathrm{GFP}^{+}$ progenitors exhibiting a short morphology and lacking a basal endfoot can be found 
253 dividing at the ventricular wall (top) and at a small distance from ventricle (bottom),

254 corresponding to respectively a short neural precursor and subapical progenitor. (G)

255 In contrast, dividing $\mathrm{T}^{2} \mathrm{1}^{-} / \mathrm{TdTomato}^{+}$cells predominantly retain a basal process

256 during division and can be found dividing at both the ventricular wall (top) and at a

257 distance from the ventricle (bottom) corresponding to respectively a radial glial cell

258 and bipolar radial glial cell. (H) Quantification of cells in the proliferative zones of

259 the LGE containing a basal process. Note that significantly more T $\alpha 1^{-} /$TdTomato $^{+}$

260 cells retain a basal process. (I) Estimation of cell cycle duration using embryonic

261 tissue fixed at variable delays after IUE and labeling for the mitotic marker phospho-

262 histone $\mathrm{H} 3(\mathrm{pH} 3)$. A rapid increase in the number of neural progenitors that were

263 positive of the marker $\mathrm{pH} 3$ was taken as a return to the mitotic ('M') phase for that

264 population of neural progenitors and a proxy for the cell cycle duration (' $\mathrm{Tc}$ ').

265

\section{Both progenitor pools can generate striatal D1 and D2 spiny projection neurons}

268 Our in utero electroporation labeling experiments suggest that $\mathrm{T} \alpha 1$ expression can

269 distinguish between two pools of neural progenitor in the LGE. The Tal-expressing

270 neural progenitors exhibit many of the characteristics of described short neural

271 precursors and subapical progenitors whereas, in contrast, the non-Ta1-expressing

272 neural progenitors tend to share many characteristics with the described radial glial

273 cells within the LGE (Pilz et al., 2013). Overall, our observations would suggest the

274 pool of Tal-expressing neural progenitors is enriched for apically dividing

275 intermediate progenitors and therefore we will refer to them collectively as apical

276 intermediate progenitors (aIPs) and the neurons they generate as aIP derived and the

277 non-T $\alpha 1$-expressing neural progenitors consist of all other neural progenitors that are

278 actively dividing at the same time and we will refer to them collectively as other

279 progenitors (OPs) and the neurons they generate as OP derived.

280 When we let the electroporated embryos develop postnatally we observe many

281 GFP and TdTomato expressing neurons spread throughout the striatum (Figure 2B).

282 The GFP expressing neurons are generated from the population of aIP neural

283 progenitors and are referred to as aIP derived and the TdTomato expressing neurons

284 from the population of OP neural progenitors and are referred to as OP derived

285 (Figure 2B). At higher magnifications both pools of labeled neurons exhibit the 286 morphology of striatal spiny projection neurons (SPNs) including dendrites densely

287 covered with spines (Figure 2B). The relative proportion of aIP and OP derived 288 neurons seen postnatally is very similar to the ratio of labeled embryonic neural 289 progenitors found 24 hours after IUE at E15.5, with a significantly larger proportion 
of OP derived neurons (aIP derived: $36.7 \pm 5.5 \%$ and OP derived: $63.3 \pm 5.5 \%$, $\mathrm{p}=0.0017$, paired t-test, $\mathrm{n}=16$ brains, Figure 2C). We next investigated the spatial distribution of aIP and OP derived neurons in rostral, central and caudal sections of

293 the striatum in a total of 15 electroporated brains (Supplemental Figure 2). In 294 general, across all sections we find that the normalised position (see Methods) of all 295 labeled SPNs was biased to the medial aspects (Wilcoxon Sign Rank, $p=0.000005, n$

$296=15$ mice $/ 23$ sections, Figure 2D), but not to either dorsal or ventral aspects of the striatum (Wilcoxon Sign Rank, $p>0.05$ ). We find that within individual sections the average location of aIP derived and OP derived neurons differ suggesting that they might exhibit different migration patterns (Halliday \& Cepko, 1992; Tan \& Breen, 1993; Reid \& Walsh, 2002; Tinterri et al., 2018). Although this likely is not captured fully in coronal slices, we nonetheless find that aIP derived neurons were approximately twice as likely to be statistically biased (Wilcoxon Sign rank, p < $0.016)$ towards the medial aspect of striatum ( $73 \%$ of sections) than OP derived neurons (37\% of sections) with the strongest medial bias seen in the central and caudal planes (Supplemental Figure 2).

We next investigated which types of striatal SPN were labeled. Combining immunocytochemistry for the marker for spiny projection neurons CTIP2 (Arlotta et al., 2008) and the marker for D2 SPNs PPE (Gerfen et al., 1990) we investigated the degree of co-localization of these markers with our aIP and OP derived striatal neurons (Figure 2E). We find that nearly all labeled neurons co-localize with CTIP2 (aIP derived/CTIP2 $2^{+}: 99.6 \pm 0.4 \%$ and OP derived/CTIP2 ${ }^{+}: 99.4 \pm 0.3 \%$, both $\mathrm{n}=9$,

312 Figure 2F) suggesting the labeled neurons are SPNs. Secondly, labeling for the D2 313 SPN marker PPE reveals that both progenitor pools can generate both $\mathrm{PPE}^{-} \mathrm{D} 1-$ 314 expressing direct pathway SPNs and $\mathrm{PPE}^{+} \mathrm{D} 2$-expressing indirect pathway SPNs (aIP 315 derived/PPE $: 35.2 \pm 7.0 \%$ and $\mathrm{OP}$ derived/PPE $: 48.2 \pm 5.3 \%, \mathrm{n}=9$ ) with a trend for 316 the aIP progenitor pool to generate more PPE ${ }^{-}$D1 SPNs (vs. 50\%, p $<0.071$, one317 sample t-test, $\mathrm{n}=8$ ). Lastly, we asked whether SPNs derived from these distinct progenitor pools end up in different compartments of the striatum. We used antibody staining against the $\mu$-opioid receptor (MOR) to delineate the MOR-rich patches and the MOR-poor matrix compartments of the striatum (Figure 2G). We find that on average only $6 \%$ of labeled neurons can be found in the striatal patches with the remainder found in the matrix with no difference between aIP and OP derived neurons (aIP/MOR $: 4.8 \pm 1.3 \%$ and $\mathrm{OP} / \mathrm{MOR}^{+}: 7.8 \pm 1.6 \%, \mathrm{n}=12$, Figure $\left.2 \mathrm{H}\right)$. As 
different embryonic stages have been shown to differently contribute to the formation of the striatal neurons and compartments (Brand \& Rakic, 1979; Graybiel \& Hickey, 1982; Marchand \& Lajoie, 1986; van der Kooy \& Fishell, 1987; Fishell et al., 1990; Newman et al., 2015; Kelly et al., 2018; Tinterri et al., 2018) we repeated these experiment using IUE at E12.5 (Supplemental Figure 3). We find broadly similar results in that the proportion of labeled adult neurons reflect the ratio of embryonic progenitors with less aIP derived neurons labeled during this earlier stage of neurogenesis (aIP derived: $6.6 \pm 4.0 \%$ and OP derived: $93.4 \pm 4.0 \%, \mathrm{n}=10$, Supplemental Figure 3B), virtually all labeled neurons express the SPN marker CTIP2 (aIP derived/CTIP2 ${ }^{+}: 100.0 \pm 0.0 \%$ and OP derived $/ \mathrm{CTIP}^{+}: 99.2 \pm 0.5 \%, \mathrm{n}=$ 6, Supplemental Figure 3C) and both progenitor populations can generate putative D1-expressing direct pathway SPNs and D2-expressing indirect pathway SPNs (aIP derived/PPE ${ }^{+}: 19.2 \pm 3.6 \%$ and OP-derived/PPE ${ }^{+}: 34.0 \pm 6.0 \% \mathrm{n}=8$, Supplemental Figure 3D). However, labeling at this younger embryonic age seems to generate fewer indirect pathway D2 SPNs (E12.5 vs. E15.5, Mann-Whitney test, $\mathrm{p}=0.037 ; \mathrm{n}=$ 8 and 12) and slightly more labeled neurons in $\mathrm{MOR}^{+}$patches (E12.5: $12.4 \pm 2.4 \%$ and E15.5: 7.0 $\pm 1.3 \%$ in $\mathrm{MOR}^{+}$patches; Mann-Whitney test, $\mathrm{p}=0.170, \mathrm{n}=4$ and 12 , Supplemental Figure 3E). Lastly, the LGE has been shown to not only generate striatal SPNs, but has also been shown to generate interneurons found in the olfactory bulb (Stenman et al., 2003). Therefore, we investigated whether we could find labeled neurons also in the olfactory bulb in a small subset of mice that had undergone IUE at E15.5. We did find small numbers of labeled neurons in sections of the olfactory bulb ( $\sim 40$ neurons per section) with the majority being OP derived (aIP derived olfactory bulb interneurons: $3.9 \pm 0.7 \%$ and OP derived olfactory bulb interneurons: $96.1 \pm 0.7$, $\mathrm{n}=5$ brains, Supplemental Figure 4).

Overall, these results suggest that aIP and OP neural progenitors in the LGE mainly generate D1 and D2 spiny projection neurons, which can be found intermingled in the striatum as well as in patch and matrix compartments. Furthermore, a small number of olfactory bulb neurons are generated mainly from the population of OP neural progenitors. 

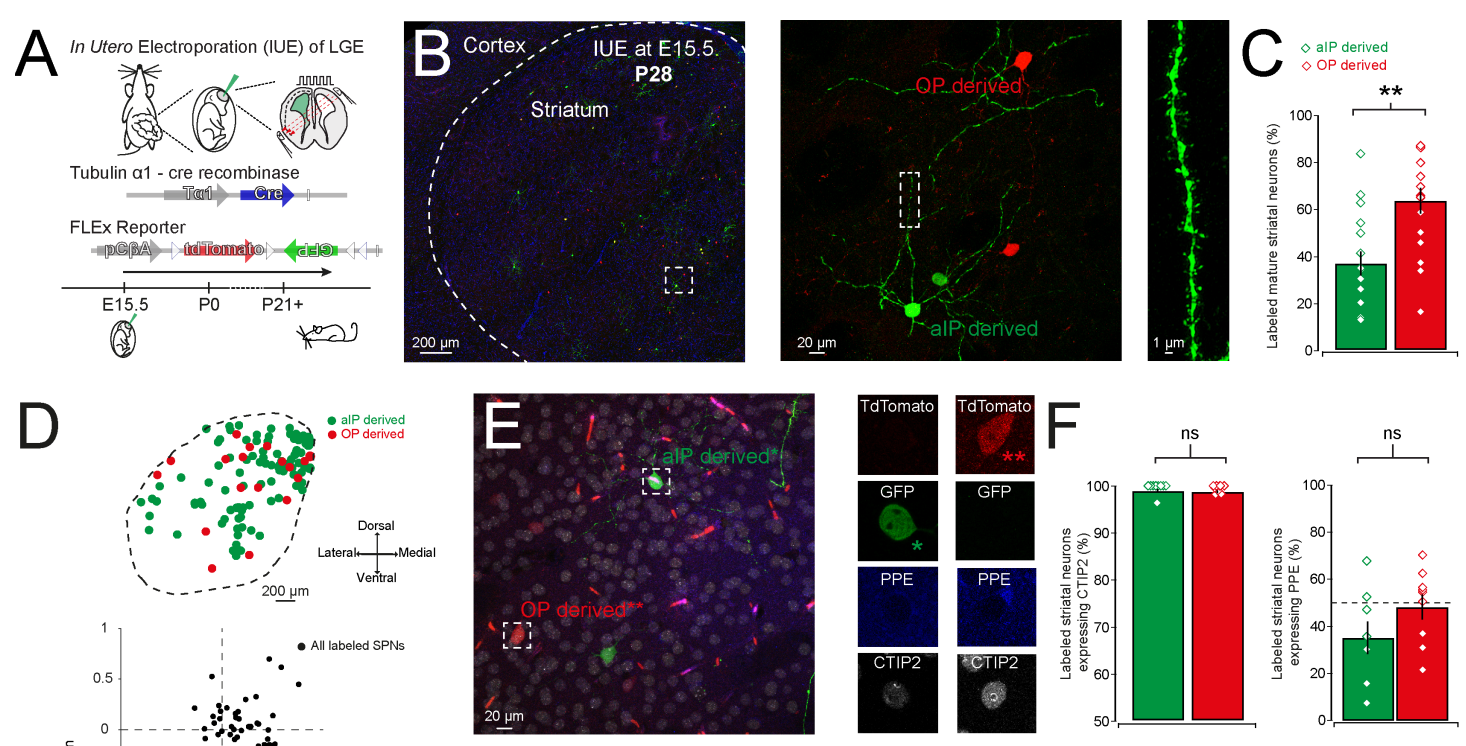

Figure 2: Both aIP and OP neural progenitors in the LGE can generate D1 and
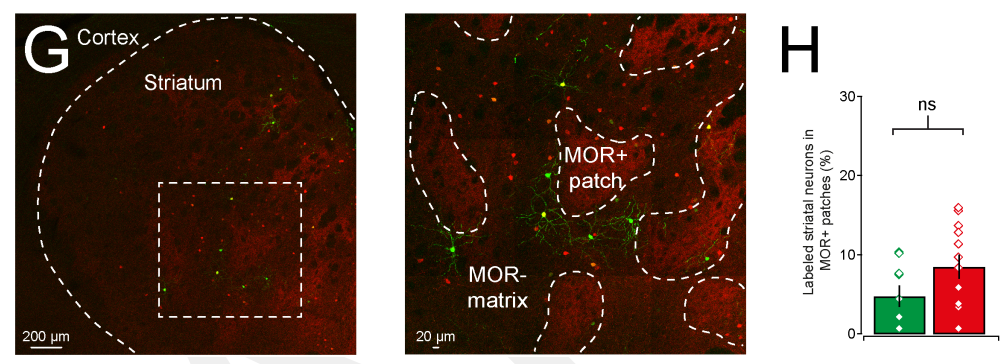

D2 striatal spiny projection neurons. (A) Mice that underwent IUE at E15.5 with Ta1-cre and FLEx reporter plasmids were left to grow up untill young adulthood (postnatal day 21 and over). (B) The striatum of these mice contain both $\mathrm{GFP}^{+}$and TdTomato $^{+}$neurons derived from respectively a pool of apical intermediate progenitors (aIPs) or other progenitors (OPs). The adult neurons derived from both progenitor pools display the radial morphology and spine studded dendrites (right) characteristic of striatal spiny projection neurons (SPNs). (C) Quantification of the relative proportion of aIP and OP derived striatal neurons reveals a similar distribution as the respective embryonic neural progenitors at E15.5. Note the significantly larger proportion of OP derived neurons. (D) Example diagram of location of aIP and OP derived neurons in a central section of the striatum (top). Normalised mediolateral and dorsoventral positions of all labeled neurons across all counted sections reveals that labeled neurons inhabit more medial aspects of the striatum (middle). Normalised mediolateral and dorsoventral positions of aIP and OP derived neurons within individual sections, linked with lines, shows that in many sections they reside in different striatal regions (bottom). (E) An example image of striatum containing aIP and OP derived neurons and labeled for the SPN marker CTIP2 (white) and the D2-SPN marker PPE (blue). The red streaks result from auto- 
380 (D2) and PPE negative (putative D1) SPNs. (G) Labeling for the $\mu$-opioid receptor 381 (MOR) reveals that aIP and OP derived neurons can be found in both $\mathrm{MOR}^{+}$patch 382 and MOR ${ }^{-}$matrix compartments. (H) Quantification of aIP and OP derived neurons 383 labeled for MOR reveals both can be found within $\mathrm{MOR}^{+}$patches in equal numbers.

Both aIP and OP derived neurons have electrical and morphological properties consistent with SPNs.

388 So far we have shown that we can pulse-label two different pools of neural 389 progenitors in the LGE and that both pools can generate D1 and D2 striatal SPNs 390 based on their neurochemical profile. We next investigated in more detail whether the 391 labeled neurons exhibit the electrophysiological and morphological properties of 392 SPNs. To do this we performed whole-cell patch-clamp recordings of aIP and OP 393 derived neurons as well as unlabeled neurons in dorsal striatum (Figure 3A). The 394 inclusion of biocytin in the intracellular solution allowed for post-hoc labeling and 395 reconstruction of recorded cells (Figure 3B and C). We investigated a variety of 396 electrophysiological properties, including membrane potential (aIP derived: $-80.3 \pm$ $3971.2 \mathrm{mV}$, OP derived: $-78.9 \pm 0.7 \mathrm{mV}$ and unlabeled: $-78.3 \pm 1.2 \mathrm{mV}$; $\mathrm{p}>0.05$, t-test, $\mathrm{n}$ $398=15,34$ and 18 neurons), input resistance (aIP derived: $88.1 \pm 8.4 \mathrm{M} \Omega$, OP derived: $39981.0 \pm 4.7 \mathrm{M} \Omega$ and unlabeled: $80.3 \pm 7.5 \mathrm{M} \Omega ; \mathrm{p}>0.05$, t-test), action potential 400 threshold (aIP derived: $-41.6 \pm 1.8 \mathrm{mV}$, OP derived: $-39.8 \pm 0.9 \mathrm{mV}$ and unlabeled: $40139.5 \pm 1.2 \mathrm{mV} ; \mathrm{p}>0.05$, t-test) as well as action potential frequency, which all 402 suggested that labeled neurons exhibited electrical properties consistent with those of 403 SPNs. However, we did not observe significant differences in these or other 404 electrophysiological properties between aIP and OP derived neurons (Figure 3D and 405 Table 1). 



$\mathrm{F}$

G
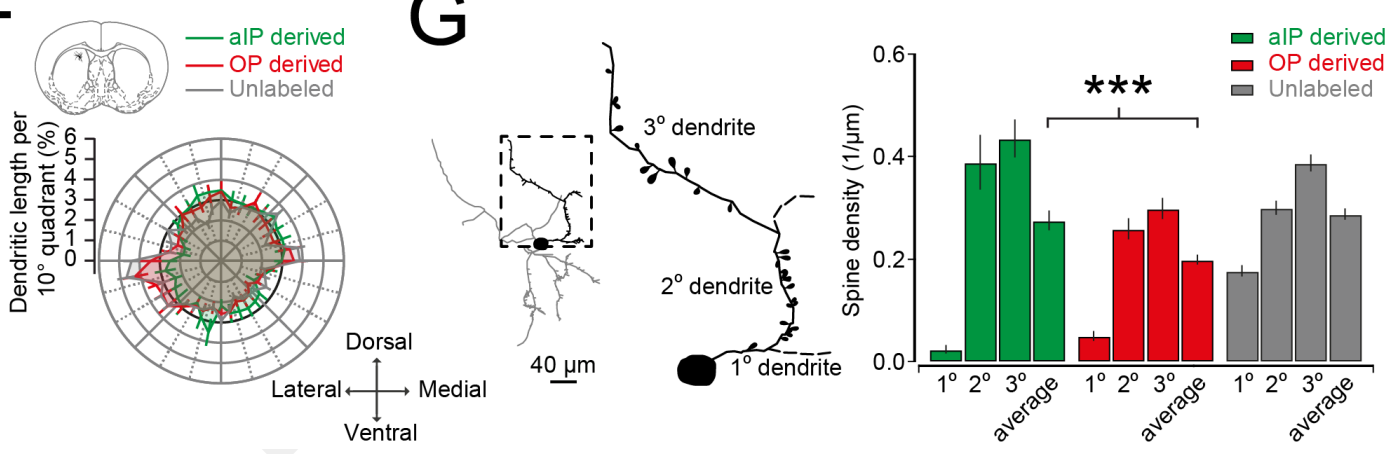

Figure 3: aIP derived SPNs exhibit greater dendritic complexity and spine density. (A) Electrophysiological properties of progenitor-derived striatal neurons were assessed using whole-cell patch-clamp recordings in acute brain slices. Example Dodt contrast image (top) and fluorescence image (bottom) of a recorded aIP derived $\mathrm{GFP}^{+}$neuron. Inset: response of the neuron to hyperpolarizing and depolarizing current steps consistent with that of a SPN. (B) Recorded cells were labeled with biocytin during recordings and revealed using streptavidin-Alexafluor405 conjugated antibodies in fixed tissue (indicated with a cross) and tested for the expression of the 
neighboring $\mathrm{CTIP}^{+}$and $\mathrm{PPE}^{+}$neurons. (C) Recorded cells were further processed for DAB immunohistochemistry allowing for reconstruction of their dendritic arbors as well as spine counts. (D) Hyperpolarizing and depolarizing current steps were used to characterise the electrophysiological properties of aIP derived, OP derived and unlabeled striatal neurons. The electrophysiological properties were consistent with those of SPNs and no significant differences were found between either group in their resting membrane potential, input resistance, spike threshold or action potential frequency. (E) Example reconstructions of DAB processed aIP derived (top), OP derived (middle) as well as unlabeled SPNs (bottom). The total dendritic length of the different SPNs did not differ. Assessment of dendritic complexity using Scholl analysis reveals that aIP derived neurons exhibit a subtle but significant larger dendritic complexity close to the soma. (F) Dendritic polarity analysis reveals that all SPNs exhibit a similar radial morphology. (G) Quantification of the number of spines on the primary $\left(1^{\circ}\right)$, secondary $\left(2^{\circ}\right)$ and tertiary $\left(3^{\circ}\right)$ dendrites reveals that aIP derived neurons exhibit an overall higher density of spines as compared to OP derived neurons.

We next investigated the morphological properties of the SPNs derived from aIP and OP progenitors. Recorded cells were post hoc processed for DAB immunohistochemistry allowing for reconstruction of their dendritic arbors as well as quantification of dendritic spines (Figure 3E - G). Comparing the total dendritic length we did not find significant differences in the length of their dendrites (aIP derived: $2184.7 \pm 228.8 \mu \mathrm{m}$, OP derived: $2299.3 \pm 147.2 \mu \mathrm{m}$ and unlabeled neurons: $2182.6 \pm 129.3 \mu \mathrm{m} ; \mathrm{p}=0.626$, Kruskal-Wallis $\mathrm{H}$ test, $\mathrm{n}=11,16$ and 35 neurons, Figure 3E). We next investigated the dendritic complexity using Scholl analysis and find that all neurons exhibit the greatest dendritic complexity close to the soma $(\sim 50$ $\mu \mathrm{m}$ distance) with a small by significant greater complexity for aIP derived over OP derived neurons $(p=0.048$, ANOVA, $n=11$ and 16, Figure 3E). Polarity analysis shows that the orientation of the dendrites is not significantly different between aIP derived, OP derived or unlabeled neurons and that all exhibit a radial morphology (Figure 3F). Quantification of dendritic spines on the primary, secondary and tertiary dendritic segments reveal that the aIP derived SPNs have a higher density of spines than the OP derived SPNs (average aIP derived: $0.28 \pm 0.02$ spines/ $\mu \mathrm{m}$ and average OP derived: $0.20 \pm 0.01$ spines/ $\mu \mathrm{m}$, t-test, $\mathrm{p}=0.0058, \mathrm{n}=13$ and 28 , Figure 3G). Combined these results suggest that both aIP and OP derived neurons exhibit all the electrical and morphological hallmarks of striatal SPNs. Furthermore, they 
455 show that subtle differences can be detected in the morphological properties of neurons derived from different progenitor pools (Guillamon-Vivancos et al., 2019) in that aIP derived neurons exhibit greater local dendritic complexity and a higher density of dendritic spines.

\section{Integration of progenitor derived striatal SPNs into striatal neural circuits}

462 We next asked to what extent aIP and OP derived neurons are integrated within the 463 circuits of the basal ganglia and striatum. To do this we performed IUE at E15.5 to

464 label both aIP derived and OP derived SPNs and examined both the synaptic inputs and outputs of labeled SPNs. We first investigated to what extent aIP and OP derived SPNs sample cortical afferents. Cortical afferents were activated using a stimulating electrode placed in the external capsule while recording from both aIP and OP derived neurons in the striatum (Figure 4B). All experiments were performed in the presence of GABA receptor antagonists to avoid erroneous activation of GABAergic afferents

470 (see Methods). We find that aIP derived and OP derived SPNs exhibit similar 471 amplitude EPSPs (aIP derived: $1.70 \pm 0.44 \mathrm{mV}$ and OP derived: $2.08 \pm 0.62 \mathrm{mV}$, $\mathrm{p}=0.974$, Mann Whitney test, $\mathrm{n}=12$ and 10, Figure 4D) and that these do not differ

473 from amplitudes found in unlabeled SPNs (unlabeled: $1.50 \pm 0.33 \mathrm{mV}$, aIP vs 474 unlabeled: $p=0.648$ and OP vs unlabeled: $p=0.650$ ). However, we find that the 475 duration and decay time of the EPSPs are significantly longer in the aIP derived

476 neurons (Table 2) suggesting they might contain different configurations of glutamate 477 receptors.

We next asked to what extent these progenitor derived neurons innervate neighbouring SPNs and in particular whether aIP derived neurons similarly innervate 480 the D1-expressing direct pathway and D2-expressing indirect pathway SPNs. We 481 used electroporation of T $\alpha 1$-cre and creON-mCherry plasmids (Saunders et al., 2012) 482 at E15.5 to selectively express the light-activatable channel ChR2 in aIP derived 483 neurons in $\mathrm{C} 57 \mathrm{Bl} / 6$ and $\mathrm{D} 1$ or D2-GFP transgenic mice with the latter facilitating the 484 classification of patched neurons as either D1 or D2 SPNs (Figure 5A). 
In Utero Electroporation (IUE) of LGE

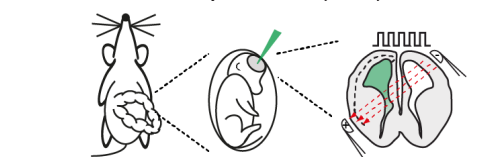

Tubulin $\alpha 1$ - cre recombinase FLEx Reporter
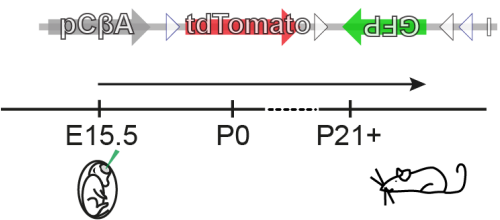

$\mathrm{B}$
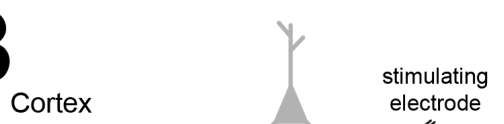

Cortex

Figure 4: Both aIP and OP derived SPNs receive cortical input. (A) IUE of Ta1Cre and FLEx reporter plasmids was performed at E15.5 to label both aIP and OP derived striatal neurons. (B) Diagram of the experimental setup consisting of electrical stimulation of cortical afferents while recording from both aIP and OP derived neurons in the striatum. All recordings were performed in the presence of GABA receptor antagonists. (C) Example Dodt contrast (left) and fluorescence (right) image of the experimental setup consisting of a Tungsten electrode placed in the external capsule and the simultaneous recording of a $\mathrm{GFP}^{+}$aIP derived SPN and a TdTomato $^{+}$OP derived SPN. (D) Scatter plot of the amplitudes of the excitatory postsynaptic responses for aIP derived, OP derived and unlabeled SPNs upon stimulation of the cortical afferents. Note that all three classes of SPN receive similar amplitude cortically evoked EPSPs.

In postnatal acute brain slices we were now able to deliver short pulses of blue light to activate ChR2-mCherry expressing aIP derived neurons (Figure 5C) while performing whole-cell voltage-clamp recordings of single or pairs of D1 and D2 SPNs at a holding potential of $0 \mathrm{mV}$ to facilitate detection of inhibitory events (Figure 5B and D). We recorded from a total of 63 SPNs of which 17 were confirmed D1 SPNs,

50815 were confirmed D2 SPNs and 31 were unclassified. Although we find that the 509 incidence of finding a connected SPN is slightly higher for the D1 SPNs than the D2 510 SPNs (D1 SPN: $47.1 \%$ and D2 SPN: 26.7\%) this does not reach statistical 
511 significance ( $\mathrm{p}=0.517$; Fisher's exact test). For the neurons that received inputs from

512 the aIP derived neurons we find no significant difference in the amplitude of the light

513 evoked inhibitory postsynaptic current (IPSC) as recorded at a holding potential of 0

$514 \mathrm{mV}(\mathrm{D} 1: 31.23 \pm 18.11 \mathrm{pA}, \mathrm{D} 2: 31.97 \pm 14.69 \mathrm{pA}$ and unclassified: $34.15 \pm 15.30$

$515 \mathrm{pA}, \mathrm{p}=0.797$, Kruskall Wallis test, $\mathrm{n}=8,4$ and 12, Figure 5E).

516 In conclusion, these results suggest that both aIP and OP derived SPNs are

517 embedded within the neural circuits of the striatum. Indeed, both aIP and OP derived

518 striatal SPNs receive excitatory glutamatergic cortical input and aIP derived neurons

519 send inhibitory GABAergic outputs to neighbouring D1 and D2 SPNs. Interestingly,

520 we find that aIP and OP derived neurons differentially respond to cortical inputs, with

521 aIP derived SPNs exhibiting prolonged EPSP kinetics, suggesting that they might

522 sample excitatory inputs differently.

In Utero Electroporation (IUE) of LGE

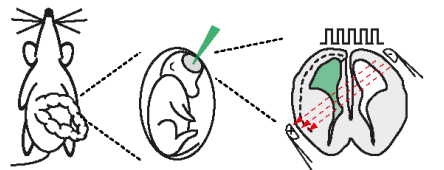

Tubulin a1 - cre recombinase

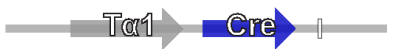

ChR2-mCherry 'Cre-On'

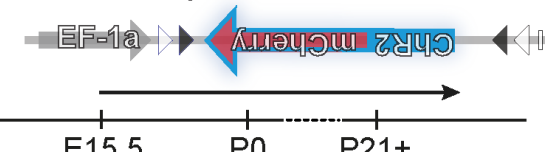

(2)

$D$
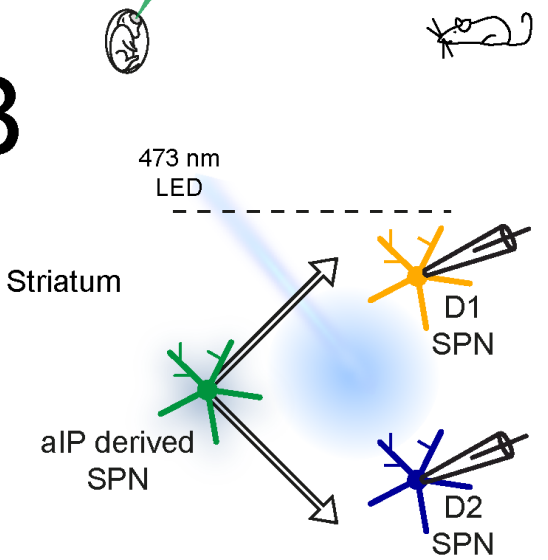
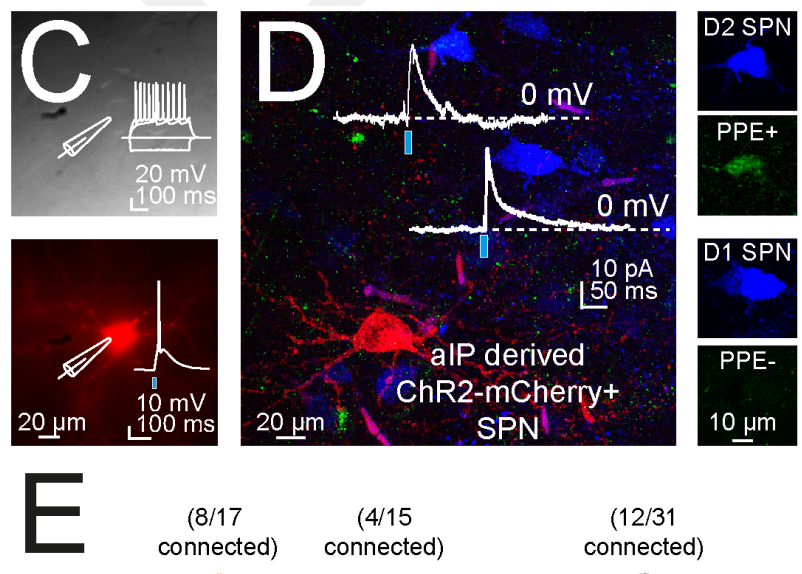

$(8 / 17$
connected)

$(4 / 15$
connected)

$(12 / 31$
connected $)$
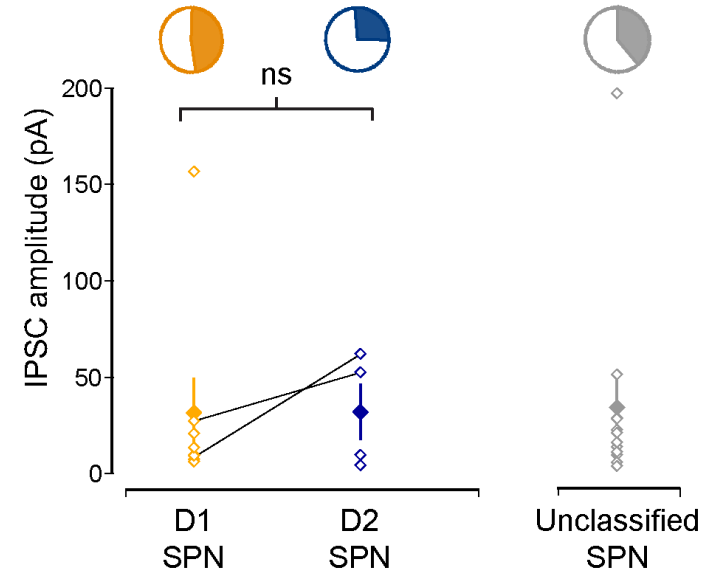

524

Figure 5: aIP derived SPNs connect to both D1 and D2 SPNs (A) IUE of T $\alpha 1$-Cre and DIO-ChR2-mCherry plasmids allowed for the expression of ChR2-mCherry in aIP derived neurons. (B) Diagram of the experimental setup consisting of recordings of D1 and D2 SPNs while stimulating aIP derived neurons with a $473 \mathrm{~nm}$ wide-field LED. All recordings were performed in the presence of glutamate receptor 
530 antagonists. (C) Whole-cell patch-clamp recordings of aIP derived ChR2-mCherry

531 expressing striatal neurons revealed that $\sim 3 \mathrm{~ms}$ flashes of light could elicit single 532 action potentials. Inset top: response of the neuron to hyperpolarising and depolarising 533 current steps. (D) To investigate striatal inhibitory synaptic output from aIP derived 534 neurons, simultaneous whole-cell patch-clamp recordings were performed from D1 535 and D2 SPNs, as determined during recordings in D1 or D2-GFP transgenic mice or 536 posthoc using immunocytochemistry for PPE. Example image of streptavidin labeled $537 \mathrm{PPE}^{+}$(upper neuron) and $\mathrm{PPE}^{-} \mathrm{SPN}$ (lower neuron) in close apposition to an aIP 538 derived-ChR2-mCherry ${ }^{+}$SPN. Traces correspond to light evoked IPSCs. (E) aIP 539 derived striatal neurons exhibited a slight preference to connect to D1 SPNs (47\%) 540 and optical activation of aIP derived neurons results in similar amplitude IPSCs in all 541 tested D1, D2 and unclassified SPNs.

\section{Discussion}

546 In this study we examined to what extent neural progenitor pool diversity in the lateral ganglionic eminence (LGE) contributes to cellular and circuit diversity in the striatum. Using in utero electroporation we are able to label two distinct pools of dividing neural progenitors in the LGE, which are active during later stages of neurogenesis. Using differential expression of the tubulin alpha1 (Ta1) promoter we are able to label a Ta1-expressing pool of neural progenitors we refer to as apical intermediate progenitors (aIPs) and a non-Ta1-expressing pool we refer to as other progenitors (OPs). We find that both progenitor types are actively dividing in the ventricular zone of the LGE around embryonic days (E)15.5-16.5, but exhibit distinct proliferative behaviours. Whereas aIP progenitors exhibit a short round morphology during division, lack a basal process and exhibit fast cell cycle kinetics, OP progenitors tend to retain a basal process and exhibit slower cell cycle kinetics. We show that both can generate D1-expressing direct pathway spiny projection neurons (SPNs) as well as D2-expressing indirect pathway SPNs found in both patch and matrix compartments of the striatum. Whereas aIP and OP derived SPNs were found intermingled throughout the striatum on average the aIP derived population of SPNs inhabited more medial aspects of the striatum. Whole-cell patch-clamp recordings and

563 morphological reconstruction of aIP and OP derived neurons confirmed they were 564 SPNs with aIP derived SPNs exhibiting a more complex local dendritic tree and an 565 increased dendritic spine density. Electrical circuit mapping shows that both aIP and 566 OP derived SPNs sample input from cortical excitatory afferents with aIP derived 
567 SPNs exhibiting differential sampling and longer duration responses. Lastly, optogenetic circuit mapping reveals that aIP derived SPNs send local GABAergic outputs to both D1 and D2 SPNs. Overall, we show that it is possible to label diverse pools of neural progenitors in the LGE and their progeny in the striatum. Diversity in

571 the pool of progenitors can generate diversity in adult striatal neurons, which 572 manifests itself in different spatial distributions, morphological properties and 573 sampling of excitatory afferents.

\section{Different pools of embryonic neural progenitors in the $L G E$}

576 The neural progenitors in the LGE of the embryonic mouse brain (Smart, 1976) 577 generate most of the SPNs of the striatum as well as some of the interneurons of the 578 olfactory bulb (Olsson et al., 1998; Stenman et al., 2003) with postnatal generation of 579 striatal neurons also contributing (Stopczynski et al., 2008). Recent studies have 580 highlighted that the LGE contains as much heterogeneity in neural progenitors (Pilz et 581 al., 2013) as pallial structures (Franco \& Muller, 2013). Indeed, in the LGE these progenitors include radial glial cells, basal radial glial cells, basal progenitors, subapical progenitors as well as short neural precursors, amongst others (Pilz et al., 2013). It is currently unknown how this heterogeneity at the progenitor level relates to the cellular and circuit diversity found in the striatum.

Several lines of evidence suggest that that the population of progenitors labeled using the promoter sequence for Ta1 correspond to the short neural precursors and subapical progenitors recently described in the LGE (Gal et al., 2006; Stancik et al., 2010; Pilz et al., 2013; Ellender et al., 2018). We find that the population of Ta1expressing neural progenitors divides at some distance from the ventricular wall but mostly with the ventricular zone, their cell cycle kinetics are fast and correspond to about 17 hours, they form a large population of dividing progenitors during later 593 stages of neurogenesis (E15.5-E16.5) and lastly they lack a basal process during 594 division. These criteria are consistent to those described for short neural precursors 595 and the subapical progenitors that are often generated from the short neural precursors (Pilz et al., 2013). Conversely, even though we find that our population of non-Ta1597 expressing neural progenitors also divides in the ventricular zone, it is more dominant 598 during early stages of neurogenesis (E12.5/E13.5), has slower cell cycle kinetics 599 corresponding to about 20 hours (Pilz et al., 2013), and many progenitors retain a 600 basal process during division. These criteria are consistent with the described 
601 morphology of radial glial cells in the LGE (Pilz et al., 2013) and the cortex (Gal et

602

603

604

605

606

607

608

609

610

611

612

613

614

615

616

617

618

619

620

621

622

623

624

625

626

627

628

629

630

631

632

633

634

al., 2006; Kriegstein \& Alvarez-Buylla, 2009).

As the Tal-expressing neural progenitors could be found actively dividing in the apical aspects of the ventricular zone and had many properties of both short neural precursors and subapical progenitors (Pilz et al., 2013) we proposed to refer to them collectively as apical intermediate progenitors (aIPs) and our non-Tal-expressing neural progenitors as other progenitors (OPs). Therefore, at the point of labeling, our in utero strategy marked a progenitor population enriched for aIPs and a population of concurrently dividing OPs. Although the method allowed us to distinguish neurons derived from different progenitor pools, it provides limited information on the lineage pathways taken by the neurons. Moreover, neural progenitor lineage in the LGE is complex with extended proliferative divisions and likely both progenitor pools generate neurons via other progenitors (Pilz et al., 2013).

\section{Progenitor derived striatal spiny projection neurons and associated circuits}

We find that both aIP and OP neural progenitors generate neurons that can be found intermingled in the striatum. By varying the embryonic time at which we perform our IUE we confirm that aIP progenitors are mainly active at later stages of neurogenesis as we find more aIP derived neurons when we electroporate at E15.5 as compared to E12.5. Immunohistochemistry for the SPN marker CTIP2 (Arlotta et al., 2008) reveals that most if not all labeled neurons are SPNs independent of whether IUE was performed at E12.5 or E15.5. Furthermore, immunohistochemistry for the D2 SPN marker PPE (Gerfen et al., 1990) reveals that both aIP and OP progenitor pools can generate D1 and D2 SPNs at both E12.5 and E15.5. These observations are consistent with the idea that the generation of D1 and D2 SPNs is controlled by other genetic factors, environmental factors or molecular clocks (Lobo et al., 2006; Franco et al., 2012; Cepko, 2014; Kelly et al., 2018). Indeed, similar observations have been made in the cortex where both layer 4 stellate neurons and layer $2 / 3$ pyramidal neurons can be generated from Tal-expressing as well as GLAST-expressing neural progenitors (Stancik et al., 2010) and indeed a single cortical progenitor can generate clones with layer-specific characteristics in all layers of cortex (Yu et al., 2009). Whereas the neural progenitor pool of origin did not seem to affect SPN type or location in patch or matrix compartments (Pert et al., 1976; Gerfen, 1984; Herkenham et al., 1984; Gerfen et al., 1985; Jimenez-Castellanos \& Graybiel, 1989; Gerfen, 1992; Crittenden 
$635 \&$ Graybiel, 2011), we find that the earlier time of electroporation resulted in both

636

637

638

639

640

641

642

643

644

645

646

647

648

649

650

651

652

653

654

655

656

657

658

659

660

661

662

663

664

665

666

667

668

pools of progenitors generating a higher proportion of D1 SPNs (Marchand \& Lajoie, 1986; van der Kooy \& Fishell, 1987; Kelly et al., 2018) as well as generating more SPNs in patch compartments (Brand \& Rakic, 1979; Graybiel \& Hickey, 1982; van der Kooy \& Fishell, 1987; Fishell et al., 1990; Newman et al., 2015; Tinterri et al., 2018).

Interestingly, we find that the different pools of neural progenitors generate D1 and D2 SPNs found in different aspects of the striatum, with aIP derived SPNs on average found in more medial aspects of the striatum. Already at embryonic ages the aIPs and related young neurons are found at slightly more distal locations relative to the ventricle, which might suggest that the location of aIP derived neurons in the adult brain results from such early differences. However, migration in the striatum is complex in that newly born neurons undergo tangential, radial and other forms of migration (Halliday \& Cepko, 1992; Tan \& Breen, 1993; Reid \& Walsh, 2002; Tinterri et al., 2018), suggesting other factors could also contribute to the ultimate location of striatal neurons (Franco et al., 2012; Kelly et al., 2018). Interestingly, detailed studies of the inputs to the striatum have revealed that dorsomedial aspects of the striatum exhibit a high degree of input heterogeneity (Pan et al., 2010; Guo et al., 2015; Hunnicutt et al., 2016) suggesting these different progenitor derived SPNs might process distinct inputs.

Electrophysiological and morphological study of single neurons reveals that both aIP and OP derived neurons mostly exhibit similar properties consistent with those of SPNs (Day et al., 2008; Gertler et al., 2008; Krajeski et al., 2018). Interestingly, we find that aIP derived neurons exhibit a greater local dendritic complexity and a higher dendritic spine density than OP derived neurons. This difference might suggest that they differentially sample excitatory inputs. Indeed, whereas the overall strength of cortical inputs to both aIP and OP derived neurons seems similar, the aIP derived neurons exhibit comparatively long duration EPSPs. This might well result from the expression of a different complement of glutamate receptors postsynaptically.

Lastly, it is known that SPNs in the striatum form strong lateral inhibitory connections with each other with which they can regulate each other's activity (Taverna et al., 2008; Planert et al., 2010; Chuhma et al., 2011; Krajeski et al., 2018) and these are not uniform but depend on the type of pre- and postsynaptic SPN 
669 (Taverna et al., 2008; Planert et al., 2010; Krajeski et al., 2018). For example, the D2

670 SPNs form a large number of strong reciprocal connections with each other (Planert et

671 al., 2010; Krajeski et al., 2018). Although our current results suggest that aIP derived

672 SPNs innervate both local D1 and D2 SPNs, future work will further inform us on

673 whether and how diverse neural progenitor pools contribute to the establishment of

674 further fine-scale neural circuits in the striatum.

675 In conclusion, we show that we are able to fluorescently pulse-label two different 676 pools of neural progenitors in the LGE and their offspring. Using this method we 677 reveal that heterogeneity in neural progenitor pools in the LGE can contribute to 678 diversity in the spiny projection neurons and circuits of the striatum. In particular, we 679 find that aspects of spatial location and morphology of neurons, as well as their 680 sampling of excitatory afferents seem particularly sensitive to neural progenitor 681 origin. Future investigations will be informative as to what extent they contribute to 682 other aspects of striatal diversity (Grillner \& Robertson, 2016) and whether neural 683 progenitor dysregulation plays a role in early onset neurodevelopmental disorders 684 such as OCD, autism and Tourette's syndrome (Graybiel \& Rauch, 2000; Del Campo 685 et al., 2011; Langen et al., 2011; McNaught \& Mink, 2011; Shepherd, 2013; Albin, 686 2018). 


\section{References}

Albin, R.L. (2018) Tourette syndrome: a disorder of the social decision-making network. Brain, 141, 332-347.

Arlotta, P., Molyneaux, B.J., Jabaudon, D., Yoshida, Y. \& Macklis, J.D. (2008) Ctip2 controls the differentiation of medium spiny neurons and the establishment of the cellular architecture of the striatum. J Neurosci, 28, 622-632.

Bagetta, V., Picconi, B., Marinucci, S., Sgobio, C., Pendolino, V., Ghiglieri, V., Fusco, F.R., Giampa, C. \& Calabresi, P. (2011) Dopamine-dependent long-term depression is expressed in striatal spiny neurons of both direct and indirect pathways: implications for Parkinson's disease. J Neurosci, 31, 12513-12522.

Baumgart, J. \& Baumgart, N. (2016) Cortex-, Hippocampus-, Thalamus-, Hypothalamus-, Lateral Septal Nucleus- and Striatum-specific In Utero Electroporation in the C57BL/6 Mouse.J Vis Exp.

Brand, S. \& Rakic, P. (1979) Genesis of the primate neostriatum: [3H]thymidine autoradiographic analysis of the time of neuron origin in the rhesus monkey. Neuroscience, 4, 767-778.

Cadwell, C.R., Scala, F., Fahey, P.G., Kobak, D., Sinz, F.H., Johnsson, P., Li, S., Cotton, R.J., Sandberg, R., Berens, P., Jiang, X. \& Tolias, A.S. (2019) Cell type composition and circuit organization of neocortical radial clones. bioRxiv, 526681.

Cepko, C. (2014) Intrinsically different retinal progenitor cells produce specific types of progeny. Nature reviews, 15, 615-627.

Chan, C.S., Peterson, J.D., Gertler, T.S., Glajch, K.E., Quintana, R.E., Cui, Q., Sebel, L.E., Plotkin, J.L., Shen, W., Heiman, M., Heintz, N., Greengard, P. \& Surmeier, D.J. (2012) Strain-specific regulation of striatal phenotype in Drd2-eGFP BAC transgenic mice. J Neurosci, 32, 9124-9132.

Chuhma, N., Tanaka, K.F., Hen, R. \& Rayport, S. (2011) Functional connectome of the striatal medium spiny neuron. J Neurosci, 31, 1183-1192.

Crittenden, J.R. \& Graybiel, A.M. (2011) Basal Ganglia disorders associated with imbalances in the striatal striosome and matrix compartments. Frontiers in neuroanatomy, $\mathbf{5}, 59$.

Day, M., Wokosin, D., Plotkin, J.L., Tian, X. \& Surmeier, D.J. (2008) Differential excitability and modulation of striatal medium spiny neuron dendrites. $J$ Neurosci, 28, 11603-11614. 
Del Campo, N., Chamberlain, S.R., Sahakian, B.J. \& Robbins, T.W. (2011) The roles of dopamine and noradrenaline in the pathophysiology and treatment of attention-deficit/hyperactivity disorder. Biological psychiatry, 69, e145157.

Ellender, T.J., Avery, S.V., Mahfooz, K., von Klemperer, A., Nixon, S.L., Buchan, M.J., van Rheede, J.J., Gatti, A., Waites, C., Newey, S.E. \& Akerman, C.J. (2018) Fine-scale excitatory cortical circuits reflect embryonic progenitor pools. bioRxiv.

Fishell, G., Rossant, J. \& van der Kooy, D. (1990) Neuronal lineages in chimeric mouse forebrain are segregated between compartments and in the rostrocaudal and radial planes. Dev Biol, 141, 70-83.

Flames, N., Pla, R., Gelman, D.M., Rubenstein, J.L., Puelles, L. \& Marin, O. (2007) Delineation of multiple subpallial progenitor domains by the combinatorial expression of transcriptional codes. J Neurosci, 27, 96829695.

Franco, S.J., Gil-Sanz, C., Martinez-Garay, I., Espinosa, A., Harkins-Perry, S.R., Ramos, C. \& Muller, U. (2012) Fate-Restricted Neural Progenitors in the Mammalian Cerebral Cortex. Science, 337, 746-749.

Franco, S.J. \& Muller, U. (2013) Shaping our minds: stem and progenitor cell diversity in the mammalian neocortex. Neuron, 77, 19-34.

Franklin, K.B.J. \& Paxinos, G. (2008) The mouse brain in stereotaxic coordinates. Elsevier Academic Press, Amsterdam ; London.

Gal, J.S., Morozov, Y.M., Ayoub, A.E., Chatterjee, M., Rakic, P. \& Haydar, T.F. (2006) Molecular and morphological heterogeneity of neural precursors in the mouse neocortical proliferative zones. J Neurosci, 26, 1045-1056.

Garas, F.N., Kormann, E., Shah, R.S., Vinciati, F., Smith, Y., Magill, P.J. \& Sharott, A. (2018) Structural and molecular heterogeneity of calretinin-expressing interneurons in the rodent and primate striatum. The Journal of comparative neurology, 526, 877-898.

Garas, F.N., Shah, R.S., Kormann, E., Doig, N.M., Vinciati, F., Nakamura, K.C., Dorst, M.C., Smith, Y., Magill, P.J. \& Sharott, A. (2016) Secretagogin expression delineates functionally-specialized populations of striatal parvalbumincontaining interneurons. Elife, $\mathbf{5}$.

Gerfen, C.R. (1984) The neostriatal mosaic: compartmentalization of corticostriatal input and striatonigral output systems. Nature, 311, 461464.

Gerfen, C.R. (1992) The neostriatal mosaic: multiple levels of compartmental organization. Trends in neurosciences, 15, 133-139. 
784

785

786

787

788

789

790

791

792

793

794

795

796

797

798

799

800

801

802

803

804

805

806

807

808

809

810

811

812

813

814

815

816

817

818

819

820

821

822

823

824

825

826

827

828

829

830

831

Gerfen, C.R., Baimbridge, K.G. \& Miller, J.J. (1985) The neostriatal mosaic: compartmental distribution of calcium-binding protein and parvalbumin in the basal ganglia of the rat and monkey. Proceedings of the National Academy of Sciences of the United States of America, 82, 8780-8784.

Gerfen, C.R., Engber, T.M., Mahan, L.C., Susel, Z., Chase, T.N., Monsma, F.J., Jr. \& Sibley, D.R. (1990) D1 and D2 dopamine receptor-regulated gene expression of striatonigral and striatopallidal neurons. Science, 250, 1429-1432.

Gertler, T.S., Chan, C.S. \& Surmeier, D.J. (2008) Dichotomous anatomical properties of adult striatal medium spiny neurons. J Neurosci, 28, 1081410824.

Gong, S., Zheng, C., Doughty, M.L., Losos, K., Didkovsky, N., Schambra, U.B., Nowak, N.J., Joyner, A., Leblanc, G., Hatten, M.E. \& Heintz, N. (2003) A gene expression atlas of the central nervous system based on bacterial artificial chromosomes. Nature, 425, 917-925.

Graybiel, A.M. \& Hickey, T.L. (1982) Chemospecificity of ontogenetic units in the striatum: demonstration by combining $[3 \mathrm{H}]$ thymidine neuronography and histochemical staining. Proceedings of the National Academy of Sciences of the United States of America, 79, 198-202.

Graybiel, A.M. \& Ragsdale, C.W., Jr. (1978) Histochemically distinct compartments in the striatum of human, monkeys, and cat demonstrated by acetylthiocholinesterase staining. Proceedings of the National Academy of Sciences of the United States of America, 75, 5723-5726.

Graybiel, A.M. \& Rauch, S.L. (2000) Toward a neurobiology of obsessivecompulsive disorder. Neuron, 28, 343-347.

Grillner, S. \& Robertson, B. (2016) The Basal Ganglia Over 500 Million Years. Current biology : $C B, 26, \mathrm{R} 1088-\mathrm{R} 1100$.

Guillamon-Vivancos, T., Tyler, W.A., Medalla, M., Chang, W.W., Okamoto, M., Haydar, T.F. \& Luebke, J.I. (2019) Distinct Neocortical Progenitor Lineages Fine-tune Neuronal Diversity in a Layer-specific Manner. Cereb Cortex, 29, 1121-1138.

Guo, Q., Wang, D., He, X., Feng, Q., Lin, R., Xu, F., Fu, L. \& Luo, M. (2015) Wholebrain mapping of inputs to projection neurons and cholinergic interneurons in the dorsal striatum. PLoS ONE, 10, e0123381.

Halliday, A.L. \& Cepko, C.L. (1992) Generation and migration of cells in the developing striatum. Neuron, 9, 15-26. 
832

833

834

835

836

837

838

839

840

841

842

843

844

845

846

847

848

849

850

851

852

853

854

855

856

857

858

859

860

861

862

863

864

865

866

867

868

869

870

871

872

873

874

875

876

877

878

879
Herkenham, M., Edley, S.M. \& Stuart, J. (1984) Cell clusters in the nucleus accumbens of the rat, and the mosaic relationship of opiate receptors, acetylcholinesterase and subcortical afferent terminations. Neuroscience, 11, 561-593.

Hunnicutt, B.J., Jongbloets, B.C., Birdsong, W.T., Gertz, K.J., Zhong, H. \& Mao, T. (2016) A comprehensive excitatory input map of the striatum reveals novel functional organization. Elife, $\mathbf{5}$.

Jimenez-Castellanos, J. \& Graybiel, A.M. (1989) Compartmental origins of striatal efferent projections in the cat. Neuroscience, 32, 297-321.

Kelly, S.M., Raudales, R., He, M., Lee, J.H., Kim, Y., Gibb, L.G., Wu, P., Matho, K., Osten, P., Graybiel, A.M. \& Huang, Z.J. (2018) Radial Glial Lineage Progression and Differential Intermediate Progenitor Amplification Underlie Striatal Compartments and Circuit Organization. Neuron, 99, 345-361 e344.

Kowalczyk, T., Pontious, A., Englund, C., Daza, R.A., Bedogni, F., Hodge, R., Attardo, A., Bell, C., Huttner, W.B. \& Hevner, R.F. (2009) Intermediate neuronal progenitors (basal progenitors) produce pyramidal-projection neurons for all layers of cerebral cortex. Cereb Cortex, 19, 2439-2450.

Krajeski, R.N., Macey-Dare, A., van Heusden, F., Ebrahimjee, F. \& Ellender, T.J. (2018) Early postnatal development of the cellular and circuit properties of striatal D1 and D2 spiny projection neurons. bioRxiv.

Kramer, P.F., Christensen, C.H., Hazelwood, L.A., Dobi, A., Bock, R., Sibley, D.R., Mateo, Y. \& Alvarez, V.A. (2011) Dopamine D2 receptor overexpression alters behavior and physiology in Drd2-EGFP mice. J Neurosci, 31, 126132.

Kriegstein, A. \& Alvarez-Buylla, A. (2009) The glial nature of embryonic and adult neural stem cells. Annual review of neuroscience, 32, 149-184.

Langen, M., Kas, M.J., Staal, W.G., van Engeland, H. \& Durston, S. (2011) The neurobiology of repetitive behavior: of mice. Neurosci Biobehav Rev, 35, 345-355.

Lee, T., Kaneko, T., Taki, K. \& Mizuno, N. (1997) Preprodynorphin-, preproenkephalin-, and preprotachykinin-expressing neurons in the rat neostriatum: an analysis by immunocytochemistry and retrograde tracing. The Journal of comparative neurology, 386, 229-244.

Lobo, M.K., Karsten, S.L., Gray, M., Geschwind, D.H. \& Yang, X.W. (2006) FACSarray profiling of striatal projection neuron subtypes in juvenile and adult mouse brains. Nature neuroscience, 9, 443-452. 
880

881

882

883

884

885

886

887

888

889

890

891

892

893

894

895

896

897

898

899

900

901

902

903

904

905

906

907

908

909

910

911

912

913

914

915

916

917

918

919

920

921

922

923

924

925

926

927

928

Marchand, R. \& Lajoie, L. (1986) Histogenesis of the striopallidal system in the rat. Neurogenesis of its neurons. Neuroscience, 17, 573-590.

Mason, H.A., Rakowiecki, S.M., Raftopoulou, M., Nery, S., Huang, Y., Gridley, T. \& Fishell, G. (2005) Notch signaling coordinates the patterning of striatal compartments. Development, 132, 4247-4258.

McNaught, K.S. \& Mink, J.W. (2011) Advances in understanding and treatment of Tourette syndrome. Nat Rev Neurol, 7, 667-676.

Nelson, A.B., Hang, G.B., Grueter, B.A., Pascoli, V., Luscher, C., Malenka, R.C. \& Kreitzer, A.C. (2012) A comparison of striatal-dependent behaviors in wild-type and hemizygous Drd1a and Drd2 BAC transgenic mice. J Neurosci, 32, 9119-9123.

Newman, H., Liu, F.C. \& Graybiel, A.M. (2015) Dynamic ordering of early generated striatal cells destined to form the striosomal compartment of the striatum. The Journal of comparative neurology, 523, 943-962.

Noctor, S.C., Flint, A.C., Weissman, T.A., Dammerman, R.S. \& Kriegstein, A.R. (2001) Neurons derived from radial glial cells establish radial units in neocortex. Nature, 409, 714-720.

Noctor, S.C., Martinez-Cerdeno, V., Ivic, L. \& Kriegstein, A.R. (2004) Cortical neurons arise in symmetric and asymmetric division zones and migrate through specific phases. Nature neuroscience, 7, 136-144.

Olsson, M., Bjorklund, A. \& Campbell, K. (1998) Early specification of striatal projection neurons and interneuronal subtypes in the lateral and medial ganglionic eminence. Neuroscience, 84, 867-876.

Pan, W.X., Mao, T. \& Dudman, J.T. (2010) Inputs to the dorsal striatum of the mouse reflect the parallel circuit architecture of the forebrain. Frontiers in neuroanatomy, 4, 147.

Pert, C.B., Kuhar, M.J. \& Snyder, S.H. (1976) Opiate receptor: autoradiographic localization in rat brain. Proceedings of the National Academy of Sciences of the United States of America, 73, 3729-3733.

Pilz, G.A., Shitamukai, A., Reillo, I., Pacary, E., Schwausch, J., Stahl, R., Ninkovic, J., Snippert, H.J., Clevers, H., Godinho, L., Guillemot, F., Borrell, V., Matsuzaki, F. \& Gotz, M. (2013) Amplification of progenitors in the mammalian telencephalon includes a new radial glial cell type. Nature communications, 4, 2125.

Planert, H., Szydlowski, S.N., Hjorth, J.J., Grillner, S. \& Silberberg, G. (2010) Dynamics of synaptic transmission between fast-spiking interneurons and striatal projection neurons of the direct and indirect pathways. $J$ Neurosci, 30, 3499-3507. 
929

930

931

932

933

934

935

936

937

938

939

940

941

942

943

944

945

946

947

948

949

950

951

952

953

954

955

956

957

958

959

960

961

962

963

964

965

966

967

968

969

970

971

972

973

974

975

976

977

Reid, C.B. \& Walsh, C.A. (2002) Evidence of common progenitors and patterns of dispersion in rat striatum and cerebral cortex. J Neurosci, 22, 4002-4014.

Saunders, A., Johnson, C.A. \& Sabatini, B.L. (2012) Novel recombinant adenoassociated viruses for Cre activated and inactivated transgene expression in neurons. Frontiers in neural circuits, 6, 47.

Schambra, U.B. \& Schambra, U.B.A.o.p.m.b. (2008) Prenatal mouse brain atlas. Springer, New York; London.

Sharott, A., Vinciati, F., Nakamura, K.C. \& Magill, P.J. (2017) A Population of Indirect Pathway Striatal Projection Neurons Is Selectively Entrained to Parkinsonian Beta Oscillations. J Neurosci, 37, 9977-9998.

Shepherd, G.M. (2013) Corticostriatal connectivity and its role in disease. Nature reviews, 14, 278-291.

Shitamukai, A., Konno, D. \& Matsuzaki, F. (2011) Oblique radial glial divisions in the developing mouse neocortex induce self-renewing progenitors outside the germinal zone that resemble primate outer subventricular zone progenitors. J Neurosci, 31, 3683-3695.

Smart, I.H. (1976) A pilot study of cell production by the ganglionic eminences of the developing mouse brain. J Anat, 121, 71-84.

Stancik, E.K., Navarro-Quiroga, I., Sellke, R. \& Haydar, T.F. (2010) Heterogeneity in ventricular zone neural precursors contributes to neuronal fate diversity in the postnatal neocortex. J Neurosci, 30, 7028-7036.

Stenman, J., Toresson, H. \& Campbell, K. (2003) Identification of two distinct progenitor populations in the lateral ganglionic eminence: implications for striatal and olfactory bulb neurogenesis. J Neurosci, 23, 167-174.

Stopczynski, R.E., Poloskey, S.L. \& Haber, S.N. (2008) Cell proliferation in the striatum during postnatal development: preferential distribution in subregions of the ventral striatum. Brain structure \& function, 213, 119127.

Tan, S.S. \& Breen, S. (1993) Radial mosaicism and tangential cell dispersion both contribute to mouse neocortical development. Nature, 362, 638-640.

Taverna, E., Gotz, M. \& Huttner, W.B. (2014) The cell biology of neurogenesis: toward an understanding of the development and evolution of the neocortex. Annu Rev Cell Dev Biol, 30, 465-502.

Taverna, S., Ilijic, E. \& Surmeier, D.J. (2008) Recurrent collateral connections of striatal medium spiny neurons are disrupted in models of Parkinson's disease. J Neurosci, 28, 5504-5512. 
Tinterri, A., Menardy, F., Diana, M.A., Lokmane, L., Keita, M., Coulpier, F., Lemoine, S., Mailhes, C., Mathieu, B., Merchan-Sala, P., Campbell, K., Gyory, I., Grosschedl, R., Popa, D. \& Garel, S. (2018) Active intermixing of indirect and direct neurons builds the striatal mosaic. Nature communications, $\mathbf{9}$, 4725 .

Tyler, W.A., Medalla, M., Guillamon-Vivancos, T., Luebke, J.I. \& Haydar, T.F. (2015) Neural precursor lineages specify distinct neocortical pyramidal neuron types. J Neurosci, 35, 6142-6152.

van der Kooy, D. \& Fishell, G. (1987) Neuronal birthdate underlies the development of striatal compartments. Brain research, 401, 155-161.

Wamsley, B. \& Fishell, G. (2017) Genetic and activity-dependent mechanisms underlying interneuron diversity. Nature reviews, 18, 299-309.

Wang, X., Tsai, J.W., LaMonica, B. \& Kriegstein, A.R. (2011) A new subtype of progenitor cell in the mouse embryonic neocortex. Nature neuroscience, 14, 555-561.

Wonders, C.P. \& Anderson, S.A. (2006) The origin and specification of cortical interneurons. Nature reviews, 7, 687-696.

Xu, Z., Liang, Q., Song, X., Zhang, Z., Lindtner, S., Li, Z., Wen, Y., Liu, G., Guo, T., Qi, D., Wang, M., Wang, C., Li, H., You, Y., Wang, X., Chen, B., Feng, H., Rubenstein, J.L. \& Yang, Z. (2018) SP8 and SP9 coordinately promote D2type medium spiny neuron production by activating Six3 expression. Development, 145.

Yu, Y.C., Bultje, R.S., Wang, X. \& Shi, S.H. (2009) Specific synapses develop preferentially among sister excitatory neurons in the neocortex. Nature, 458, 501-504.

Yu, Y.C., He, S., Chen, S., Fu, Y., Brown, K.N., Yao, X.H., Ma, J., Gao, K.P., Sosinsky, G.E., Huang, K. \& Shi, S.H. (2012) Preferential electrical coupling regulates neocortical lineage-dependent microcircuit assembly. Nature, 486, 113117. 
1019

1020

1021

1022

1023

1024

1025

1026

1027

1028

1029

1030

1031

1032

1033

1034

1035

1036

1037

1038

1039

1040

1041

1042

1043

1044

1045

1046

1047

1048

1049

1050

1051

1052

1053

1054

1055

1056

1057

1058

1059

1060

1061

1062

\section{Methods \& Materials}

Animals

All experiments were carried out on C57/BL6 wildtype and heterozygous D1-GFP or D2-GFP mice of both sexes with ad libitum access to food and water. The D1-GFP or D2-GFP BAC transgenic mice report subtypes of the dopamine receptor, either D1 or D2, by the presence of GFP (Mutant Mouse Regional Resource Centers, MMRRC). Details of the mice and the methods of BAC mice production have been published (Gong et al., 2003) and can be found on the GENSAT website [GENSAT (2009) The Gene Expression Nervous System Atlas (GENSAT) Project. In: NINDS, Contracts N01NS02331 and HHSN271200723701C, The Rockefeller University (New York), http://www.gensat.org/index.html]. The BAC transgenic mice were backcrossed to a C57/BL6 background over 20+ generations prior to use and kept as a heterozygous mouse line to avoid published issues using these transgenic lines (Bagetta et al., 2011; Kramer et al., 2011; Chan et al., 2012; Nelson et al., 2012). All mice were bred, IVC housed in a temperature controlled animal facility (normal 12:12 h light/dark cycles) and used in accordance with the UK Animals (Scientific Procedures) Act (1986). Females were checked for plugs daily; the day of the plug was considered embryonic day (E)0.5 and injection of plasmids and in utero electroporation (IUE) was performed at E12.5 - E16.5.

\section{Electroporation}

In utero electroporation (IUE) was performed using standard procedures. In short, pregnant females were anaesthetized using isoflurane and their uterine horns were exposed by midline laparotomy. A mixture of plasmid DNA $(\sim 1.5 \mu \mathrm{g} / \mu \mathrm{l})$ and $0.03 \%$ fast green dye was injected intraventricularly using pulled micropipettes through the uterine wall and amniotic sac. Plasmid DNA included: (i) 'T $\alpha 1-C r e$ ', in which the gene for Cre recombinase is under the control of a portion of the T $\alpha 1$ promoter (Stancik et al., 2010); (ii) 'C $\beta$ A-FLEx' which uses the chicken $\beta$-actin promoter to control a flexible excision (FLEx) cassette in which Cre recombination switches expression from TdTomato fluorescent protein to enhanced green fluorescent protein (Franco et al., 2012); and (iii) 'DIO-ChR2-mCherry' (pAAV-EF1a-doublefloxedhChR2(H134R)-mCherry-WPRE-HGHpA; Addgene \#20297), in which Cre recombination turns on the expression of channelrhodopsin-2 (ChR2) under the control of the human elongation factor-1a promoter (Saunders et al., 2012). Total volume injected per pup was $\sim 1 \mu 1$. T $\alpha 1$-Cre and C $\beta$ A-FLEx constructs (and other combinations of constructs) were injected as a 1:1 ratio of plasmid DNA (each 3 $\mu \mathrm{g} / \mu \mathrm{l}$, so final concentration of both constructs was $1.5 \mu \mathrm{g} / \mu \mathrm{l})$. The cathode of the Tweezertrode (Genetronics) was placed just above the eye outside the uterine muscle and the anode was placed slightly lower at the contralateral cheek region (Baumgart $\&$ Baumgart, 2016). Five pulses (50 ms duration separated by $200-950 \mathrm{~ms}$ ) at $42-$ $60 \mathrm{~V}$ with a BTX ECM 830 pulse generator (Genetronics). Typically around $80 \%$ of the pups underwent electroporation. Afterwards the uterine horns were placed back inside the abdomen, the cavity filled with warm physiological saline and the 
1063 abdominal muscle and skin incisions were closed with vicryl and prolene sutures, 1064 respectively. Dams were placed back in a clean cage and monitored closely until the 1065 birth of the pups.

Slice preparation and recording conditions

1068

Acute striatal slices were made from postnatal animals at 3-5 weeks of age. Animals were anaesthetized with isoflurane and then decapitated. Coronal 350-400 $\mu \mathrm{m}$ slices were cut using a vibrating microtome (Microm HM650V). Slices were prepared in artificial cerebrospinal fluid (aCSF) containing (in $\mathrm{mM}$ ): 65 Sucrose, $85 \mathrm{NaCl}, 2.5$ $\mathrm{KCl}, 1.25 \mathrm{NaH}_{2} \mathrm{PO}_{4}, 7 \mathrm{MgCl}_{2}, 0.5 \mathrm{CaCl}_{2}, 25 \mathrm{NaHCO}_{3}$ and 10 glucose, $\mathrm{pH}$ 7.2-7.4, bubbled with carbogen gas $\left(95 \% \mathrm{O}_{2} / 5 \% \mathrm{CO}_{2}\right)$. Slices were immediately transferred to a storage chamber containing aCSF (in mM): $130 \mathrm{NaCl}, 3.5 \mathrm{KCl}, 1.2 \mathrm{NaH}_{2} \mathrm{PO}_{4}, 2$ $\mathrm{MgCl}_{2}, 2 \mathrm{CaCl}_{2}, 24 \mathrm{NaHCO}_{3}$ and 10 glucose, $\mathrm{pH} 7.2-7.4$, at $32{ }^{\circ} \mathrm{C}$ and bubbled with carbogen gas until used for recording. Striatal slices were transferred to a recording chamber and continuously superfused with aCSF bubbled with carbogen gas with the same composition as the storage solution $\left(32{ }^{\circ} \mathrm{C}\right.$ and perfusion speed of $2 \mathrm{ml} / \mathrm{min}$ ). Whole-cell recordings were performed using glass pipettes $(\sim 6 \mathrm{M} \Omega)$, pulled from standard wall borosilicate glass capillaries and containing for whole-cell currentclamp (in $\mathrm{mM}$ ): 110 potassium gluconate, 40 HEPES, 2 ATP-Mg, 0.3 Na-GTP, 4 $\mathrm{NaCl}$ and $4 \mathrm{mg} / \mathrm{ml}$ biocytin ( $\mathrm{pH}$ 7.2-7.3; osmolarity, 290-300 mosmol/1) and for whole-cell voltage-clamp (in $\mathrm{mM}$ ): 120 cesium gluconate, $40 \mathrm{HEPES}, 4 \mathrm{NaCl}, 2$ ATP-Mg, 0.3 Na-GTP, 0.2 QX-314 and 4 mg/ml biocytin (pH 7.2-7.3; osmolarity, 290-300 mosmol/L). Recordings were made using Multiclamp 700B amplifiers and filtered at $4 \mathrm{kHz}$ and acquired using an InstruTECH ITC-18 analog/digital board and WinWCP software (University of Strathclyde, RRID:SCR_014713) at $10 \mathrm{kHz}$.

\section{Stimulation and recording protocols}

Hyperpolarizing and depolarizing current steps were used to assess the intrinsic properties of the recorded SPNs including input resistance and spike threshold (using small incremental current steps) as well as the properties of action potentials (amplitude, frequency and duration). Properties were assessed immediately on breakin. Activation of excitatory cortical afferents was performed via a bipolar stimulating electrode (FHC Inc., USA) placed in the external capsule, and in the presence of blockers of inhibitory GABAergic transmission including the $\mathrm{GABA}_{\mathrm{A}}$-receptor antagonist SR95531 $(1 \mu \mathrm{M})$ and the GABA $_{B}$-receptor antagonist CGP52432 (2 $\left.\mu \mathrm{M}\right)$. Afferents were activated every $5 \mathrm{~s}$ with up to 20 repetitions and excitatory postsynaptic potentials (EPSPs) were recorded from the patched SPNs. Photoactivation of ChR2 was achieved using widefield 2-3 ms duration light pulses of $\sim 1 \mathrm{~mW}$ via a TTL triggered CoolLED pE-300 system (CoolLED, Andover, UK)

\section{Analysis of recordings}

Data were analyzed offline using custom written programs in Igor Pro (Wavemetrics, RRID:SCR_000325). The input resistance was calculated from the observed membrane potential change after hyperpolarizing the membrane potential with a set 
1107

1108

1109

1110

1111

1112

1113

1114

1115

1116

1117

1118

1119

1120

1121

1122

1123

1124

1125

1126

1127

1128

1129

1130

1131

1132

1133

1134

1135

1136

1137

1138

1139

1140

1141

1142

1143

1144

1145

1146

1147

1148

1149

current injection. The spike threshold was the membrane voltage at which the SPN generated an action potential. The action potential amplitude was taken from the peak amplitude of the individual action potentials relative to the average steady-state membrane depolarization during positive current injection. Action potential duration was taken as the duration between the upward and downward stroke of the action potential at $25 \%$ of the peak amplitude. Evoked EPSPs and IPSCs were defined as upward or downward deflections of more than 2 standard deviations (SD) on average synaptic responses generated after filtering and averaging original traces $(0.1 \mathrm{~Hz}$ highpass filter and $500 \mathrm{~Hz}$ low-pass filter) and used for analysis of synaptic properties. Synaptic properties include measurements of peak amplitude, duration (measured from the start of the upward/downward stroke of the event until its return to the preevent baseline), rise time (time between $20 \%$ and $80 \%$ of the peak amplitude) and decay time (measured as the time from peak amplitude until the event returned to $50 \%$ of peak amplitude).

\section{Histological analyses}

Following whole-cell patch-clamp recordings the brain slices were fixed in $4 \%$ paraformaldehyde in $0.1 \mathrm{M}$ phosphate buffer ( $\mathrm{PB}$; pH 7.4). Biocytin-filled neurons were visualized by incubating sections in 1:10,000 streptavidin AlexaFluor405conjugated antibodies (ThermoFisher Scientific, Cat\#:S32351). Visualized neurons were labeled for chicken ovalbumin upstream promoter transcription-factor interacting protein-2 (CTIP2, 1:1000, rat, Abcam, Cat\#:ab14865, RRID:AB_2064130) and pre-proenkephalin (PPE, 1:1000, rabbit, LifeSpan Biosciences, Cat\#:LS-C23084, RRID:AB_902714) in PBS containing 0.3\% Triton X-

100 (PBS-Tx) overnight at $4^{\circ} \mathrm{C}$ followed by incubation with goat-anti-rat AlexaFluor647 (1:500; ThermoFisher Scientific, CAT\#:A-21247, RRID:AB_141778) and goat-anti-rabbit AlexaFluor555 (1:500; ThermoFisher Scientific, CAT\#:A-21429, RRID:AB_2535850) or goat-anti-rabbit AlexaFluor488 (1:500; ThermoFisher Scientific, CAT\#:A32731, RRID:AB_2633280) secondary antibodies in 0.3\% PBSTx for $2 \mathrm{~h}$ at RT for D1 or D2 SPN classification. Occasionally the endogenous fluorescence would be boosted with antibodies against GFP (1:1000, chicken, Aves Labs, CAT\# GFP-1020, RRID:AB_10000240) or TdTomato (1:1000; rat; anti-RFP; Chromotek, CAT\# 5f8-100, RRID:AB_2336064) or slices were co-stained with the nuclear marker 4',6-diamidino-2-phenylindole (DAPI) in PBS $(1: 100,000)$ to facilitate the delineation of brain structures. CTIP2 is expressed by SPNs and not interneurons (Arlotta et al., 2008) and PPE reliably labels indirect pathway D2 SPNs (Lee et al., 1997; Sharott et al., 2017). PPE antibody staining was facilitated through antigen retrieval by heating sections at $80^{\circ} \mathrm{C}$ in $10 \mathrm{mM}$ sodium citrate $(\mathrm{pH}$ 6.0) for approximately 30-60 min prior to incubation with PPE primary antibody. After classification of SPNs the slices were washed 3 times in PBS and processed for DAB immunohistochemistry using standard procedures.

Whole-brain fixation of embryonic and adult brains was performed by rapid decapitation of the head and submersion in oxygenated sucrose cutting solution before 
1150 submersion in $4 \%$ paraformaldehyde in $0.1 \mathrm{M}$ phosphate buffer (PB; $\mathrm{pH} 7.4)$. The

1151 brains were fixed for $24-48$ hours, after which they were washed in PBS. Whole1152 brain tissue was directly, or in the case of embryonic tissue after embedding in 5\% 1153 agar, sectioned at $50 \mu \mathrm{m}$ on a vibrating microtome (VT1000S; Leica Microsystems). 1154 All sections were pre-incubated in 10-20\% normal donkey serum (NDS; Vector 1155 Laboratories) or normal goat serum (NGS; Vector Laboratories) in PBS for more than $11561 \mathrm{~h}$ at RT. $\mathrm{GFP}^{+}\left(\mathrm{T} \alpha 1^{+}\right)$and $\mathrm{TdTomato}^{+}\left(\mathrm{T} \alpha 1^{-}\right)$progenitors and neurons were often 1157 visualized without antibody-mediated augmentation of fluorescence but in rare cases 1158 the endogenous fluorescence was boosted with antibodies against GFP $(1: 1000$, 1159 chicken, Aves Labs, CAT\#:GFP-1020, RRID:AB_10000240) or TdTomato (1:1000; 1160 rat; anti-RFP; Chromotek, CAT\#:5f8-100, RRID:AB_2336064) and Goat-Anti1161 chicken AlexaFluor488 (1:500; Life Technologies, CAT\#:A11039, 1162 RRID:AB_142924) and Goat-Anti-rat AlexaFluor555 (1:500; Life Technologies, 1163 CAT\#:A-21429, RRID:AB_2535850). In embryonic tissue antibody labeling was 1164 used to label $\mathrm{pH} 3$ in neural progenitors (1:500; rabbit; Millipore, CAT\#:06-570, 1165 RRID:AB_310177). Adult tissue was either co-stained in 1:100,000 DAPI in PBS to 1166 facilitate the delineation of brain structures or was labeled for MOR (1:3000, goat, 1167 ImmunoStar CAT\#:24216, RRID:AB_572251) or CTIP2 (1:1000, rat: Abcam 1168 Cat\#:ab14865, RRID:AB_2064130) and PPE (1:1000, rabbit, LifeSpan Biosciences 1169 Cat\#:LS-C23084, RRID:AB_902714). PPE staining was facilitated through antigen 1170 retrieval by heating sections at $80^{\circ} \mathrm{C}$ in $10 \mathrm{mM}$ sodium citrate $(\mathrm{pH}$ 6.0) for 1171 approximately $30 \mathrm{~min}$ prior to incubation with 1:1000 rabbit anti-PPE in PBS-Tx and $11721 \%$ NDS overnight at $4^{\circ} \mathrm{C}$, after which the reaction was revealed by incubating with 1173 1:500 donkey-anti-rabbit AlexaFluor647 (1:500, Life Technologies CAT\#:A31573, 1174 RRID:AB_2536183) in PBS-Tx for $2 \mathrm{~h}$ at RT.

Stereology and analysis of tissue

1177 Fluorescence images were captured with a LSM 710 confocal microscope using ZEN 1178 software (Zeiss, RRID:SCR_013672) or Leica DM5000B epifluorescence microscope 1179 using Openlab software (PerkinElmer, RRID:SCR_012158). Counting of labeled $1180 \mathrm{GFP}^{+}$and $\mathrm{TdTomato}^{+}$progenitors and young neurons and assessing their location 1181 within the embryonic brain was performed using ImageJ (RRID:SCR_003070) on z1182 stacks of $\sim 40 \mu \mathrm{m}$ thickness. In embryonic tissue occasionally yellow cells could be 1183 seen which were counted as $\mathrm{GFP}^{+}$and were assumed to have undergone 1184 recombination relatively recently. Positive cells had a fluorescence signal that was at 1185 least twice the background fluorescence (measured from randomly selected regions of 1186 the tissue). X-and y-coordinates labeled cells were used to calculate the distance from 1187 the ventricle and spread. Counting of progenitor cell basal processes was performed in 1188 Z-stack projections of confocal stacks of $\sim 40 \mu \mathrm{m}$ thickness. All clearly delineated 1189 processes above the SVZ and extending towards the pial surface were counted. M1190 phase reentry after IUE for aIP and OP was estimated from co-labeling of cells with 1191 the mitotic marker $\mathrm{pH} 3$ in tissue fixed with varying time-delays after IUE of T $\alpha 1-\mathrm{Cre}$ 1192 and CßA-FLEx plasmids (Stancik et al., 2010). Localizing $\mathrm{GFP}^{+}$and TdTomato ${ }^{+}$ 
progenitors and young neurons in various sub regions of the LGE was performed using a combination of anatomical landmarks (Schambra \& Schambra, 2008) and previous delineations (Flames et al., 2007). Olfactory bulb analysis was performed using a total of 5 brains and all GFP or TdTomato positive cells were counted in zstacks of $\sim 40 \mu \mathrm{m}$ thickness.

Progenitor derived neuron counting and analysis was performed similar to (Garas et al., 2016; Garas et al., 2018). In brief, a version of design-based stereology, the 'modified optical fractionator' was used to generate unbiased cell counts and map distributions of striatal neurons in rostral, middle and caudal sections (Franklin \& Paxinos, 2008). Once the chosen striatal coronal planes were identified and the immunofluorescence protocol carried out, the dorsal striatum was delineated using a Zeiss Imager M2 epifluorescence microscope (Carl Zeiss, AxioImager.M2) equipped with a 20X (Numerical Aperture $=0.8$ ) objective and StereoInvestigator v9.0 software (MBF Biosciences). Imaging was subsequently performed by capturing a series of completely tessellated, z-stacked images (each $1 \mu \mathrm{m}$ thick) at depths from 2 to $12 \mu \mathrm{m}$ from the upper surface of each section at the level of the striatum (thereby defining a $10 \mu \mathrm{m}$-thick optical disector). As counts were performed across the entirety of the dorsal striatum within a given rostro-caudal plane, the grid size and counting frame were set to the same size of $420 \times 320 \mathrm{~mm}$. To minimize confounds arising from surface irregularities, neuropil within a $2 \mu \mathrm{m}$ 'guard zone' at the upper surface was not imaged. A neuron was counted if the top of its nucleus came into focus within the disector. If the nucleus was already in focus at the top of the $10 \mu \mathrm{m}$-thick optical disector the neuron was excluded. Normalised positions were calculated as described in (Garas et al., 2016; Garas et al., 2018). Mediolateral and dorsoventral bias within each individual section was assessed by computing a Wilcoxon Sign rank test on the positions of all neurons across or within groups to test whether they significantly differed from zero (minimum 8 neurons for a given section). Mediolateral and dorsoventral positions of red and green neurons across animals were compared by computing a Wilcoxon sign rank test on the normalised position in each direction for each section, when there were a minimum of 8 neurons of each type in a single section ( $\mathrm{n}=20$ sections).

DAB-immunoreactive neurons were visualized on a brightfield microscope and were reconstructed and analyzed using Neurolucida and Neuroexplorer software (MBF Bioscience, RRID:SCR_001775). Only labeled neurons that exhibited a full dendritic arbor were included for analysis e.g. cells with clear truncations were not included in the dataset. Scholl analysis and polarity analysis was performed using standard procedures. In brief, both Scholl and polarity plots were generated for individual SPNs by calculating the total dendritic length located within $10^{\circ}$ segments with increasing distance from the soma. The dendritic lengths were subsequently normalised for an individual SPN and averaging the normalised plots of individual neurons generated final plots. \\ $1235 \quad$ Statistics}


1236 All data are presented as means \pm SEM. The ' $n$ ' refers to the number of brains (Figure 12371 and 2 ) or neurons (Figure $3-5$ ) tested. Statistical tests were all two-tailed and 1238 performed using SPSS 17.0 (IBM SPSS statistics, RRID:SCR_002865) or GraphPad 1239 Prism version 5.0 (GraphPad software, RRID:SCR_002798). Synaptic connectivity 1240 ratios were compared with Fisher's Exact test. Continuous data were assessed for 1241 normality and appropriate parametric (ANOVA, paired t-test and unpaired t-test) or 1242 non-parametric (Mann-Whitney $U$ ) statistical tests were applied $\left({ }^{*} \mathrm{p}<0.05,{ }^{* *} \mathrm{p}<0.01\right.$, $1243 * * * \mathrm{p}<0.001)$. 
bioRxiv preprint doi: https://doi.org/10.1101/770057; this version posted September 25, 2019. The copyright holder for this preprint (which was not certified by peer review) is the author/funder, who has granted bioRxiv a license to display the preprint in perpetuity. It is made available under aCC-BY-ND 4.0 International license.

1246

1247

1248

1249

1250

1251

1252

1253

1254

1255

1256

1257

1258

1259

1260

1261

1262

1263

1264

1265

1266

1267

1268

1269

1270

1271

1272

1273

\section{Tables}

Table 1: Intrinsic membrane properties of progenitor derived SPNs

\begin{tabular}{|c|c|c|c|c|}
\hline & alP derived & OP derived & p-value & Unlabeled \\
\hline Resting membrane potential $(\mathrm{mV})$ & $-80.25 \pm 1.21$ & $-79.10 \pm 0.64$ & 0.28 & $-79.35 \pm 0.69$ \\
\hline Input resistance $(\mathrm{M} \Omega)$ & $88.12 \pm 6.77$ & $80.96 \pm 4.72$ & 0.36 & $80.27 \pm 7.53$ \\
\hline Spike threshold (mV) & $-40.93 \pm 1.66$ & $-39.98 \pm 0.92$ & 0.32 & $-39.47 \pm 1.22$ \\
\hline Spike rate $(500 \mathrm{pA})(\mathrm{Hz})$ & $34.17 \pm 2.09$ & $30.80 \pm 1.95$ & 0.15 & $31.46 \pm 2.42$ \\
\hline Spike rate $(400 \mathrm{pA})(\mathrm{Hz})$ & $28.58 \pm 1.75$ & $28.25 \pm 1.76$ & 0.91 & $27.57 \pm 2.14$ \\
\hline Spike rate $(300 \mathrm{pA})(\mathrm{Hz})$ & $22.31 \pm 2.71$ & $23.23 \pm 1.68$ & 0.77 & $22.50 \pm 2.13$ \\
\hline Spike rate $(200 \mathrm{pA})(\mathrm{Hz})$ & $14.75 \pm 2.19$ & $19.70 \pm 2.20$ & 0.20 & $17.78 \pm 2.65$ \\
\hline Spike rate $(100 \mathrm{pA})(\mathrm{Hz})$ & $4.17 \pm 0.83$ & $13.00 \pm 2.29$ & 0.06 & $9.17 \pm 0.83$ \\
\hline First ISI (ms) & $26.02 \pm 2.99$ & $26.92 \pm 5.04$ & 0.88 & $31.46 \pm 4.97$ \\
\hline Second ISI (ms) & $28.08 \pm 2.22$ & $27.64 \pm 1.88$ & 0.89 & $32.92 \pm 3.89$ \\
\hline Third ISI (ms) & $32.74 \pm 2.63$ & $32.85 \pm 2.26$ & 0.98 & $33.50 \pm 2.90$ \\
\hline Fourth ISI (ms) & $34.09 \pm 2.56$ & $35.17 \pm 2.36$ & 0.78 & $34.98 \pm 2.61$ \\
\hline First spike amplitude (mV) & $87.11 \pm 3.81$ & $85.76 \pm 2.76$ & 0.78 & $94.13 \pm 2.53$ \\
\hline Second spike amplitude (mV) & $73.13 \pm 4.79$ & $65.54 \pm 3.42$ & 0.22 & $81.33 \pm 3.34$ \\
\hline First spike duration (ms) & $2.17 \pm 0.21$ & $1.90 \pm 0.09$ & 0.18 & $1.69 \pm 0.13$ \\
\hline Second spike duration (ms) & $2.68 \pm 0.28$ & $2.54 \pm 0.18$ & 0.67 & $2.09 \pm 0.17$ \\
\hline
\end{tabular}

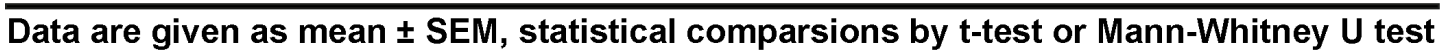


1279

1280

1281

1282

1283

Table 2: Cortical synaptic response properties

\begin{tabular}{|c|c|c|c|c|}
\hline & aIP derived & OP derived & p-value & Unlabeled \\
\hline Amplitude (pA) & $1.70 \pm 0.44$ & $2.08 \pm 0.62$ & 0.77 & $1.50 \pm 0.33$ \\
\hline Duration (ms) & $143.9 \pm 14.48$ & $99.30 \pm 10.47$ & 0.03 & $112.81 \pm 11.76$ \\
\hline Rise time (ms) & $5.73 \pm 0.42$ & $5.87 \pm 0.95$ & 0.83 & $4.47 \pm 0.29$ \\
\hline Decay time (ms) & $66.20 \pm 6.98$ & $44.63 \pm 5.32$ & 0.03 & $55.86 \pm 5.90$ \\
\hline
\end{tabular}

Data are given as mean \pm SEM, statistical comparsions by Mann-Whitney test. 
1285

1286

1287

1288

1289

1290

1291

1292

1293

1294

1295

1296

1297

1298

1299

1300

1301

1302

1303

1304

1305

1306

1307

1308

1309

1310

1311

1312

1313

1314

1315

1316

1317

1318

1319
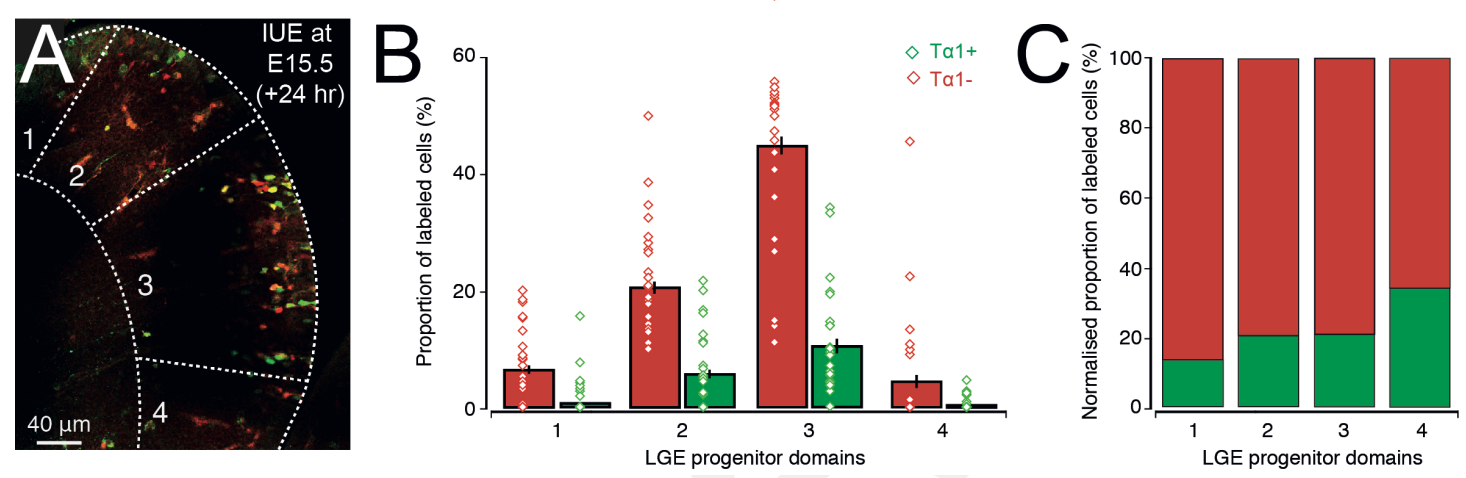

Supplementary Figure 1: IUE labeled cells can be found in all domains of the

LGE. (A) Example coronal section of an embryonic mouse brain 24 hours after IUE at E15.5 with Ta1-cre and FLEx plasmids. Note that $\mathrm{GFP}^{+}$and $\mathrm{TdTomato}^{+}$labeled cells can be seen in all progenitor domains of the LGE. (B) Average barplot of the

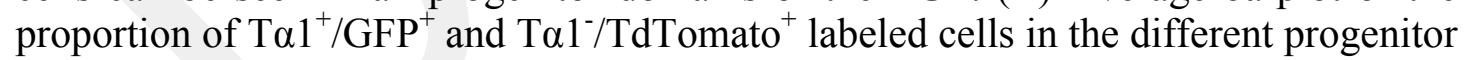
domains of the LGE. Note that our labeling strategy predominantly targets LGE3 (all labeled neurons: LGE1: 7.6\%, LGE2: 32.0\%, LGE3: 59.0\% and LGE4: 1.3\%) and that each progenitor domain of the LGE contains both $\mathrm{T} \alpha 1^{+} / \mathrm{GFP}^{+}$and $\mathrm{T}^{2} 1^{-}$ TdTomato $^{+}$progenitors. (C) The relative proportion of $\mathrm{T}_{\alpha} 1^{+} / \mathrm{GFP}^{+}$and $\mathrm{T} \alpha 1^{-}$ $/$ TdTomato $^{+}$labeled cells appears relatively constant between the different domains of the LGE. 
Rostral

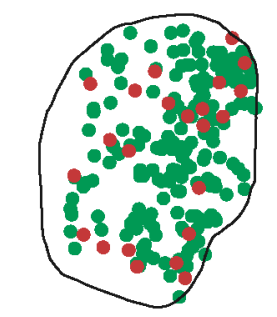

Central

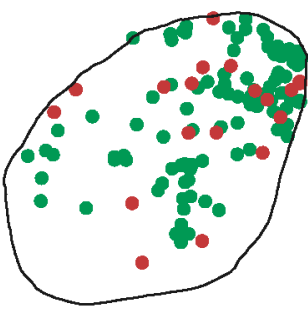

Caudal

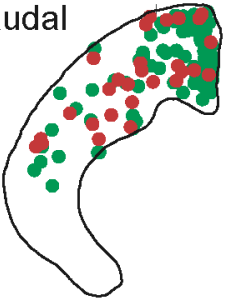

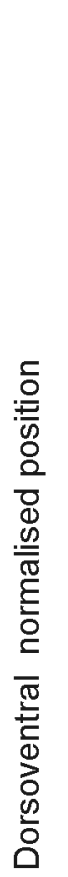


Mediolateral

Normalised

Position

Supplementary Figure 2: Labeled aIP and OP derived striatal neurons in rostral, central and caudal striatum. Left column, positions of aIP and OP derived neurons in rostral, central and caudal sections of a single animal. Right column, normalised positions of aIP and OP derived neurons in each plane (rostral $n=13$, central $\mathrm{n}=11$, caudal $\mathrm{n}=4$ mice). 

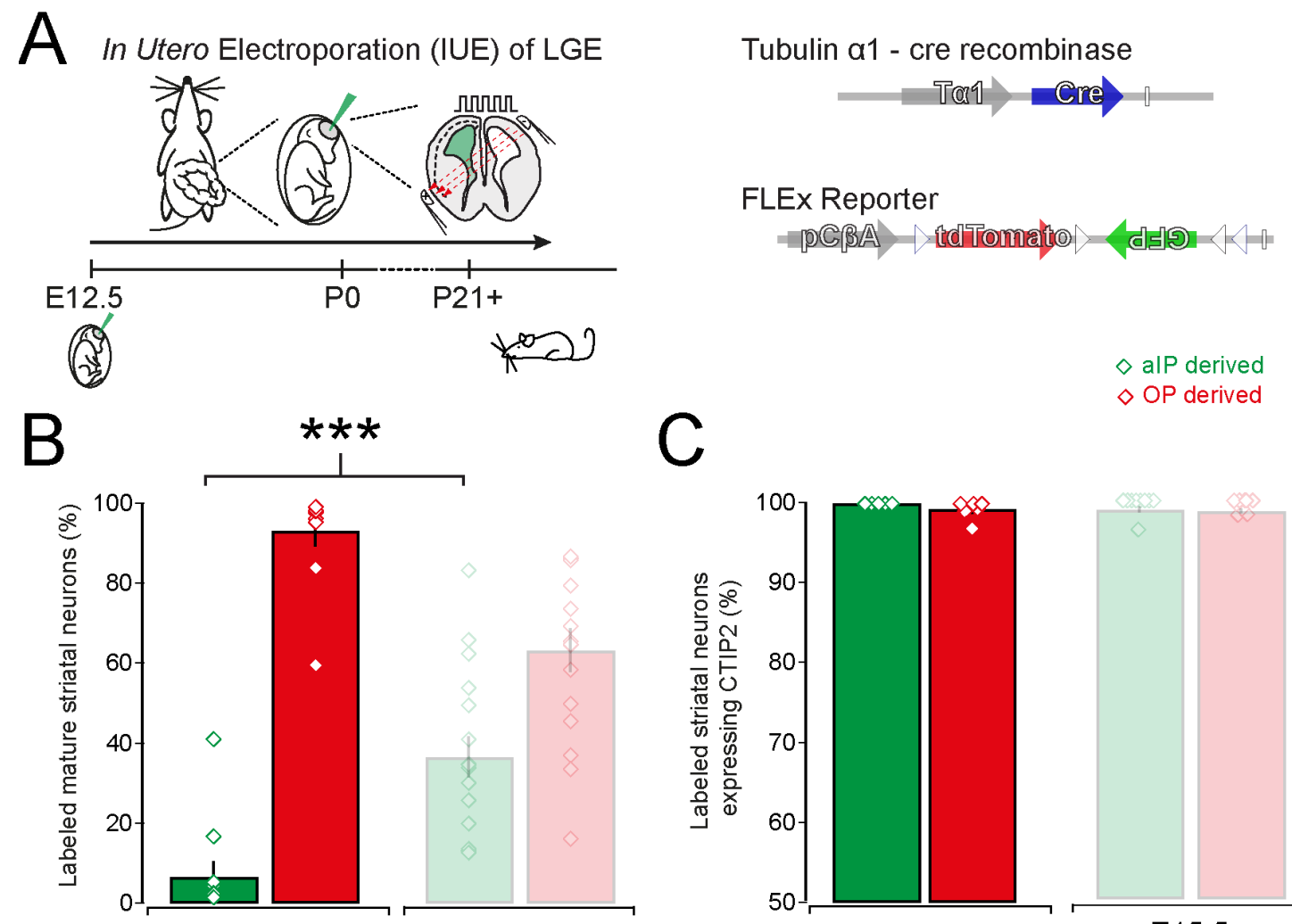

$\diamond$ alP derived
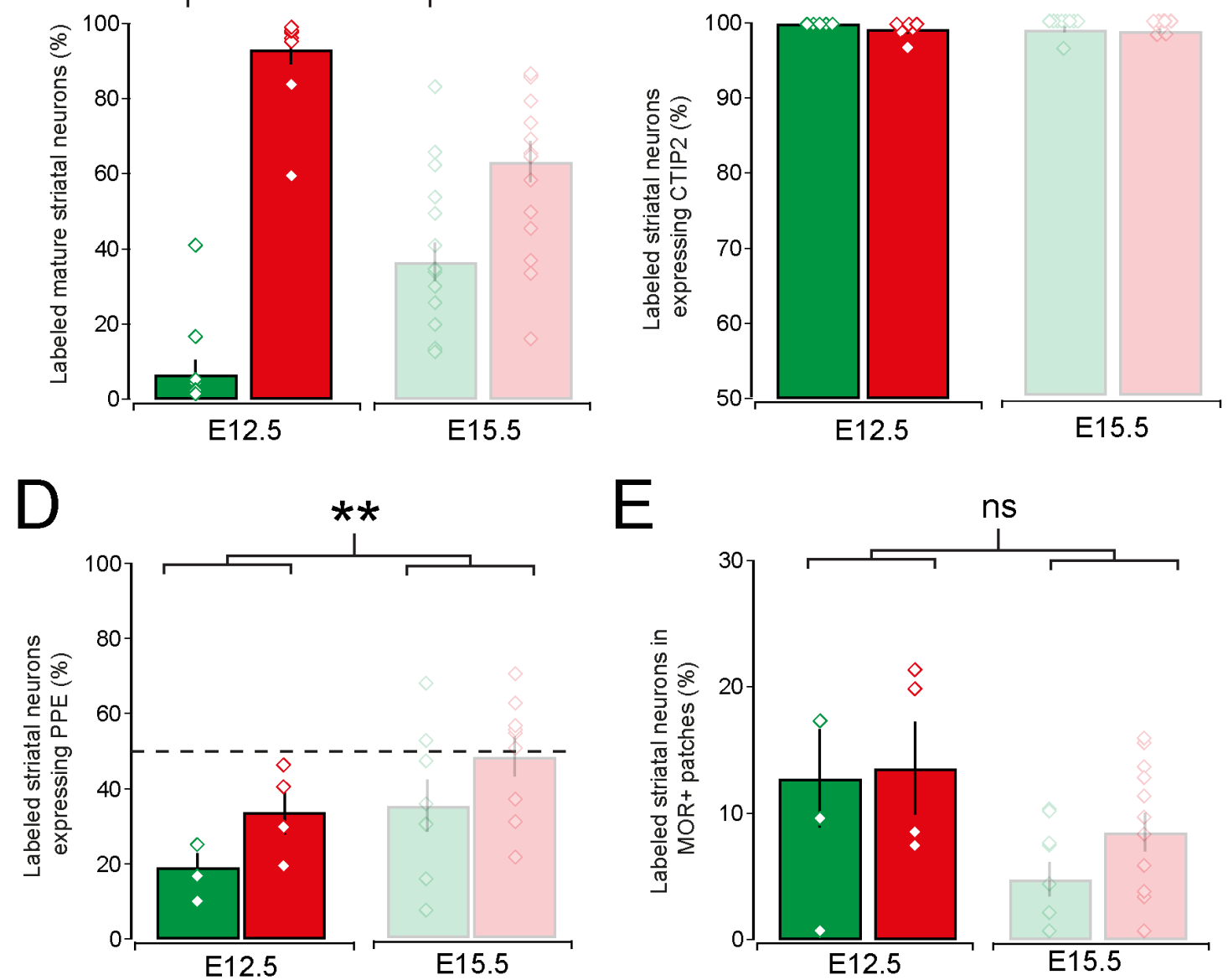

Supplementary Figure 3: Labeled aIP and OP derived striatal neurons after IUE during early stages of neurogenesis. (A) Animals that underwent IUE at E12.5 with Ta1-cre and FLEx reporter plasmids were left to mature untill young adulthood $(\mathrm{P} 21+)(\mathbf{B})$ Significantly more aIP derived neurons are generated using IUE at E15.5 than at E12.5 (E12.5: $6.6 \pm 4.0 \%$ and E15.5: $36.7 \pm 5.5 \%$, Mann-Whitney test, $\mathrm{p}=0.0002, \mathrm{n}=10$ and 16 brains). (C) IUE at both E12.5 and E15.5 generate mostly striatal CTIP $^{+}$neurons. (D) There is no significant difference in the production of $\mathrm{PPE}^{+} / \mathrm{D} 2$ SPNs by aIP or OP progenitors at E12.5 or E15.5. However, grouping all labeled neurons together at E12.5 a smaller proportion of $\mathrm{PPE}^{+} / \mathrm{D} 2 \mathrm{SPNs}$ is labeled at this early stage of neurogenesis (E12.5 vs. E15.5, Mann-Whitney test; $\mathrm{p}=0.006 ; \mathrm{n}=$ 4 and 9 brains). (E) There is a trend towards more labeled neurons in the patch compartments after IUE at E12.5 (E12.5: $12.4 \pm 2.4 \%$ vs. E15.5: $6.9 \pm 1.3 \%$ in MOR+ patches; Mann-Whitney test, $\mathrm{p}=0.170, \mathrm{n}=4$ and 12). 
bioRxiv preprint doi: https://doi.org/10.1101/770057; this version posted September 25, 2019. The copyright holder for this preprint (which was not certified by peer review) is the author/funder, who has granted bioRxiv a license to display the preprint in perpetuity. It is made available under aCC-BY-ND 4.0 International license.

1383

1384

1385

1386

1387

1388

1389

1390

1391

1392

1393

1394

1395

1396

1397

1398

1399

1400

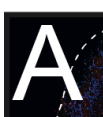

1401

1402

1403

1404

1405

1406

1407

1408

1409

1410

1411

1412
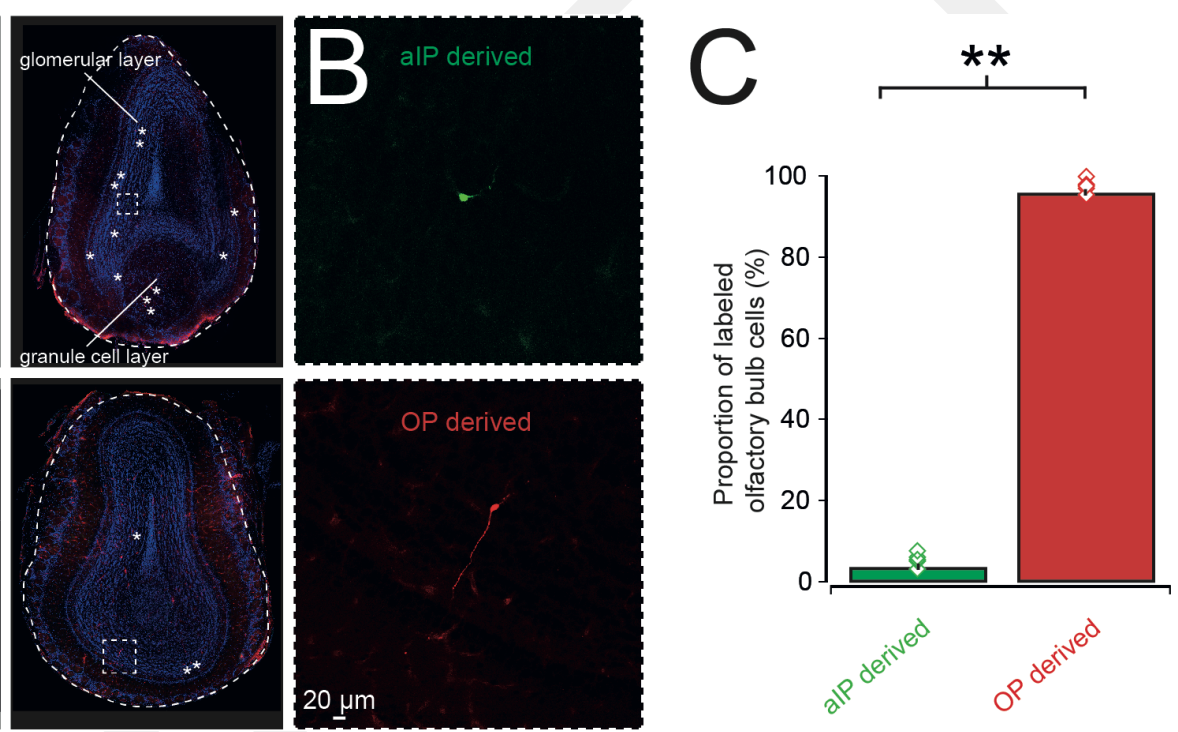

Supplementary Figure 4: IUE of LGE neural progenitors also labels a small number of olfactory bulb cells. (A) Example images of 4 different olfactory bulbs with labeled neurons indicated by asterisks. (B) High magnification image of an aIP derived cell (top) and an OP derived cell (bottom) in the olfactory bulb. (C) The majority of labeled cells in the olfactory bulb are OP derived (aIP derived: $3.9 \pm 1.6 \%$ and OP derived: $96.1 \pm 1.6 \%, p=0.0079$, Mann-Whitney test, $n=5$ brains). 